\title{
26. EOLIAN SEDIMENTATION IN THE NORTHWEST PACIFIC OCEAN: A PRELIMINARY EXAMINATION OF THE DATA FROM DEEP SEA DRILLING PROJECT SITES 576 AND 5781
}

\author{
Thomas R. Janecek, Lamont-Doherty Geological Observatory of Columbia University ${ }^{2}$
}

\begin{abstract}
The mass-accumulation rate and grain size of the total eolian component of North Pacific pelagic clays at Deep Sea Drilling Project Sites 576 and 578 have been used to evaluate changes in eolian sedimentation and the intensity of atmospheric circulation that have occurred during the past $70 \mathrm{~m}$.y. Eolian deposition, an indicator of source area aridity, was low in the Paleocene, Eocene, and Oligocene, apparently reflecting the humid environments of that time as well as the lack of glacial erosion products. A general increase in eolian accumulation in the Miocene apparently reflects the relative increase in global aridity during the latter part of the Cenozoic. A dramatic increase in eolian accumulation rates in the Pliocene reflects the increased aridity and availability of glacial erosion products associated with Northern Hemisphere glaciation 2.5 m.y. ago. Eolian grain size, an indicator of wind intensity, suggests that Late Cretaceous wind strength was comparable to present-day wind strength. A sharp decrease in eolian grain size across the Paleocene/Eocene boundary is not readily interpreted, but may indicate a significant reduction in the intensity of atmospheric circulation at that time. Fine eolian grain size and low accumulation rates in the Eocene and early Oligocene are in agreement with low early Tertiary thermal gradients and less vigorous atmospheric circulation. Large increases in grain size during the Oligocene, mid-to-late Miocene, and Pliocene appear to be a response to steepening thermal gradients resulting from increasing polar isolation.
\end{abstract}

\section{INTRODUCTION}

\section{Background and Objectives}

One of the objectives of Leg 86 of the Deep Sea Drilling Project (DSDP) was to recover a long-term record of North Pacific pelagic sedimentation in order to determine the Late Cretaceous and Cenozoic history of eolian input in the region. Until recently, the only continuous record of Cenozoic eolian deposition in the North Pacific was that of LL44-GPC-3 (Leinen and Heath, 1981; Janecek and Rea, 1983), located in the northeastern Pacific (Fig. 1). The recovery of an essentially continuous Cenozoic section at Site 576 (Figs. 1 and 2), at a similar latitude to LL44-GPC-3, provides an opportunity to evaluate differences in the accumulation rate and grain size of dust with distance of transport across the North Pacific. If plate movement and changing source areas can be accounted for, then the eolian records can be interpreted in terms of a paleoclimatic signal.

In addition to the long-term record of Site 576, a thick late Neogene siliceous clay section was recovered at Site 578 (Figs. 1 and 2). The high sedimentation rates at this site provide an opportunity to evaluate high frequency changes in terrigenous (eolian) input in the northwestern Pacific Ocean during the late Cenozoic.

\section{Eolian Sedimentation}

Nonbiogenic pelagic sediments recovered seaward of the realm of hemipelagic sedimentation and away from turbidites and ice-rafted debris are composed primarily of terrigenous material (mostly quartz and clays) trans-

\footnotetext{
${ }^{1}$ Heath, G. R., Burckle, L. H., et al., Init. Repts. DSDP, 86: Washington (U.S. Govt. Printing Office)

2 Address: Lamont-Doherty Geological Observatory of Columbia University, Palisades, NY 10964.
}

ported to the sea surface by wind. Rapid settling of these particles from surface waters by fecal pellets and large amorphous aggregates (Honjo, 1980, 1983) minimizes surface current smearing of atmospheric input patterns and thus sedimentary patterns mimic surface input patterns (Berger, 1976).

The distribution of eolian material in surface sediments has been shown to reflect present-day atmospheric circulation patterns (Rex and Goldberg, 1958; MolinaCruz and Price, 1977; Kolla et al., 1979; Thiede, 1979; Leinen and Heath, 1981). Variations in the distribution, accumulation rate, and grain size of eolian material in Pleistocene sediments reflect changes in wind intensity, latitudinal position of the zonal winds, and continental climate (Kolla et al., 1979; Pisias and Leinen, 1984; Janecek and Rea, in press).

The flux of wind-borne dust to the oceans depends, in part, on the strength of the zonal winds, on distance to source, and on the annual number of dust-transporting episodes, but is mainly dependent upon the climate of the source area (Prospero and Nees, 1977; Gillette, 1981; Rea and Janecek, 1981a). Humid climates tend to promote vegetation, thereby reducing the amount of material available for wind erosion, transport, and subsequent deposition in the deep sea. High precipitation rates also increase the scavenging of dust by rain thus decreasing the amount of deposition downwind (Windom, 1975, Parkin and Padgham, 1975). Therefore, variations in the accumulation rate of eolian material will primarily reflect changes in continental climate.

Both air-sampling (Johnson, 1976; Glaccum and Prospero, 1980) and theoretical calculations (Gillette et al., 1974; Schutz, 1979) suggest that beyond $1000 \mathrm{~km}$ distance from the source, eolian material is in equilibrium with the transporting wind. That is, the settling velocities of the particles are some small fraction of the verti- 


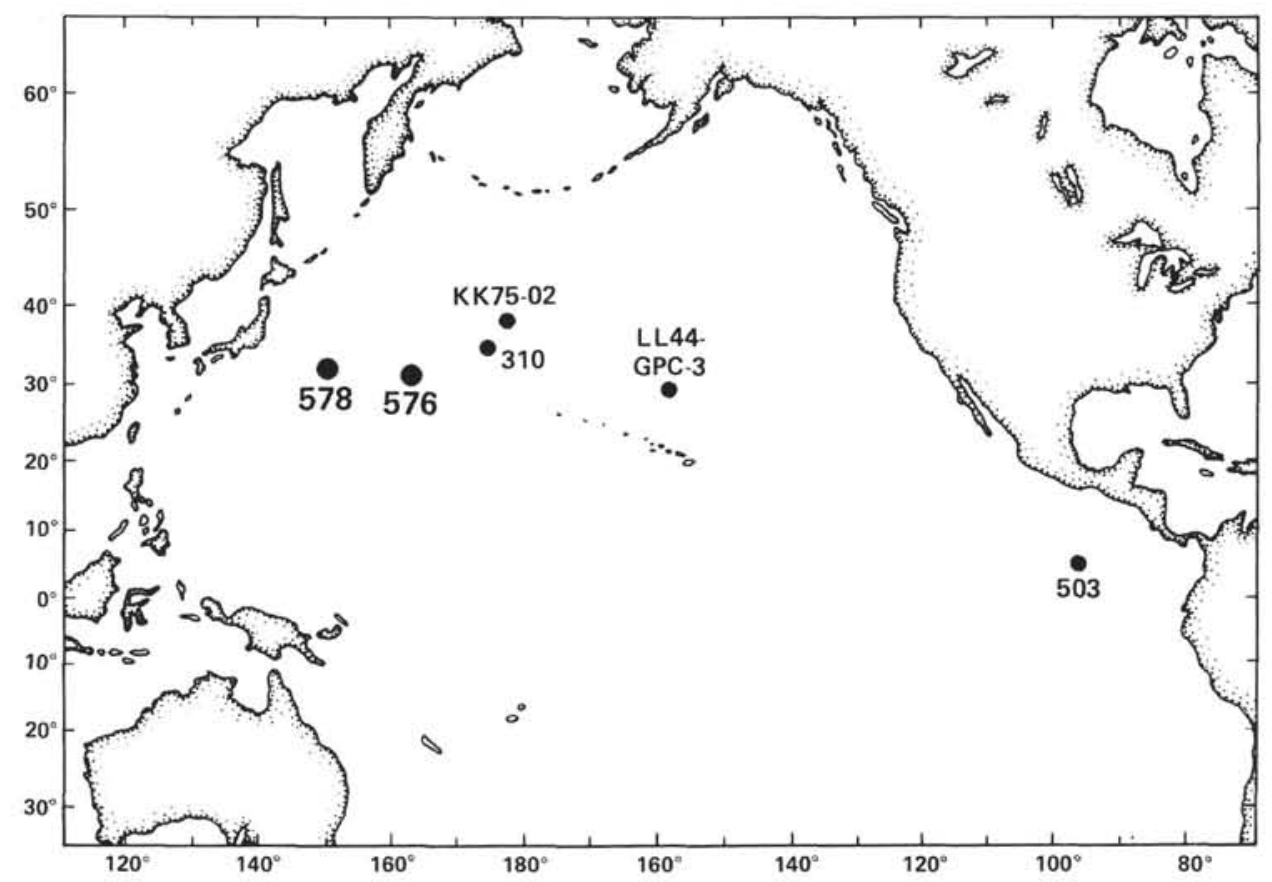

Figure 1. Location map showing DSDP Sites 578 and 576. DSDP Sites 503 and 310 (Janecek, 1983) and piston cores KK75-02 (Janecek and Rea, in press) and LL44-GPC-3 (Janecek and Rea, 1983) are also shown for reference.

cal velocity component of atmospheric motions. These equilibrium grains, which can remain in suspension indefinitely (Gillette et al., 1974), are carried global distances in the upper troposphere (Windom, 1969; Schutz, 1979) and are generally removed by rain (Jackson et al., 1973). A change in the size of the equilibrium grain represents a change in the intensity of the transporting wind, with stronger winds carrying larger particles (Parkin, 1974). Thus, the independent nature of the grain-size and accumulation-rate data allow us to determine the intensity of past atmospheric circulation and the amount of dust input to the seafloor from the same samples.

\section{Geologic Setting}

Site 576 was hydraulically piston cored to a depth of $74 \mathrm{~m}$ sub-bottom east of the Shatsky Rise. The site lies in over $6200 \mathrm{~m}$ of water beneath the central Pacific water mass (Fig. 1). Site 576 was chosen to be in an area of uniform pelagic "red clay" sedimentation between the Shatsky Rise and the Emperor Seamounts, but far enough east to minimize the fraction of the section occupied by ash layers. The area around Site 576 is characterized by gently rolling topography with less than $50 \mathrm{~m}$ of local relief (see Site 576 chapter, this volume). Three major lithologic units are recognized at Site $\mathbf{5 7 6}$ and are shown in Figure 2. Basal lithologic Unit III, consisting of chert and porcellanite fragments, forms the prominent reflector at the base of the "transparent layer" in the North Pacific.

Site 578 was drilled to $176.8 \mathrm{~m}$ sub-bottom in $6010 \mathrm{~m}$ of water west of the Shatsky Rise (Fig. 1) in an area of gently rolling seafloor with local relief of tens of meters. The seafloor is underlain by a section several hundred meters thick lying above Cretaceous chert and chalk and characterized by continuous parallel sub-bottom reflec- tors. The late Neogene section was divided into four lithologic units (Fig. 2; see Site 578 chapter, this volume).

\section{METHODS}

The samples for Site 576 were taken primarily from Hole $576 \mathrm{~B}$ at approximately $1.5-\mathrm{m}$ intervals (one per section) to a depth of $58 \mathrm{~m}$. Coring gaps in Hole 576B were filled with samples from Hole 576 (Table 1). Samples for Site 578 were taken at 30 - to 50 -cm intervals to a depth of $100 \mathrm{~m}$ (Table 2).

The basic data set for this study includes (1) the weight percent of the total eolian load rather than just the weight percent of quartz, which comprises only 20 to $40 \%$ or less of the total eolian load (Windom, 1969; Prospero and Bonatti, 1969; Johnson, 1979; Prospero et al., 1981) and (2) the grain size of the eolian material. The detrital mineral or eolian component is isolated by a series of selective extractions to remove calcium carbonate, opaline silica, Fe and Mn oxides, and hydroxides and zeolites. The amount of residual eolian material, predominantly fine-grained quartz and clays, determines the eolian weight percentage (see Rea and Janecek, $1981 \mathrm{~b}$ for details of the chemical procedures).

Grain-size analysis of the eolian component was carried out on the 6- to $10-\phi(16$ to $1 \mu \mathrm{m})$ size fraction at $0.5-\phi$ intervals using a Coulter Counter model $\mathrm{Zb}$ particle-size analyzer. This procedure is described in detail by Graham and Rea (1980), and the precision of the resulting size determinations is $\pm 0.03 \phi$ (average of difference between duplicate samples).

\section{Linear Sedimentation Rates}

At Site 576, paleomagnetic reversal stratigraphy (see Heath, Rea, and Levi, this volume) was used to calculate sedimentation rates from $25 \mathrm{~m}$ sub-bottom to the 

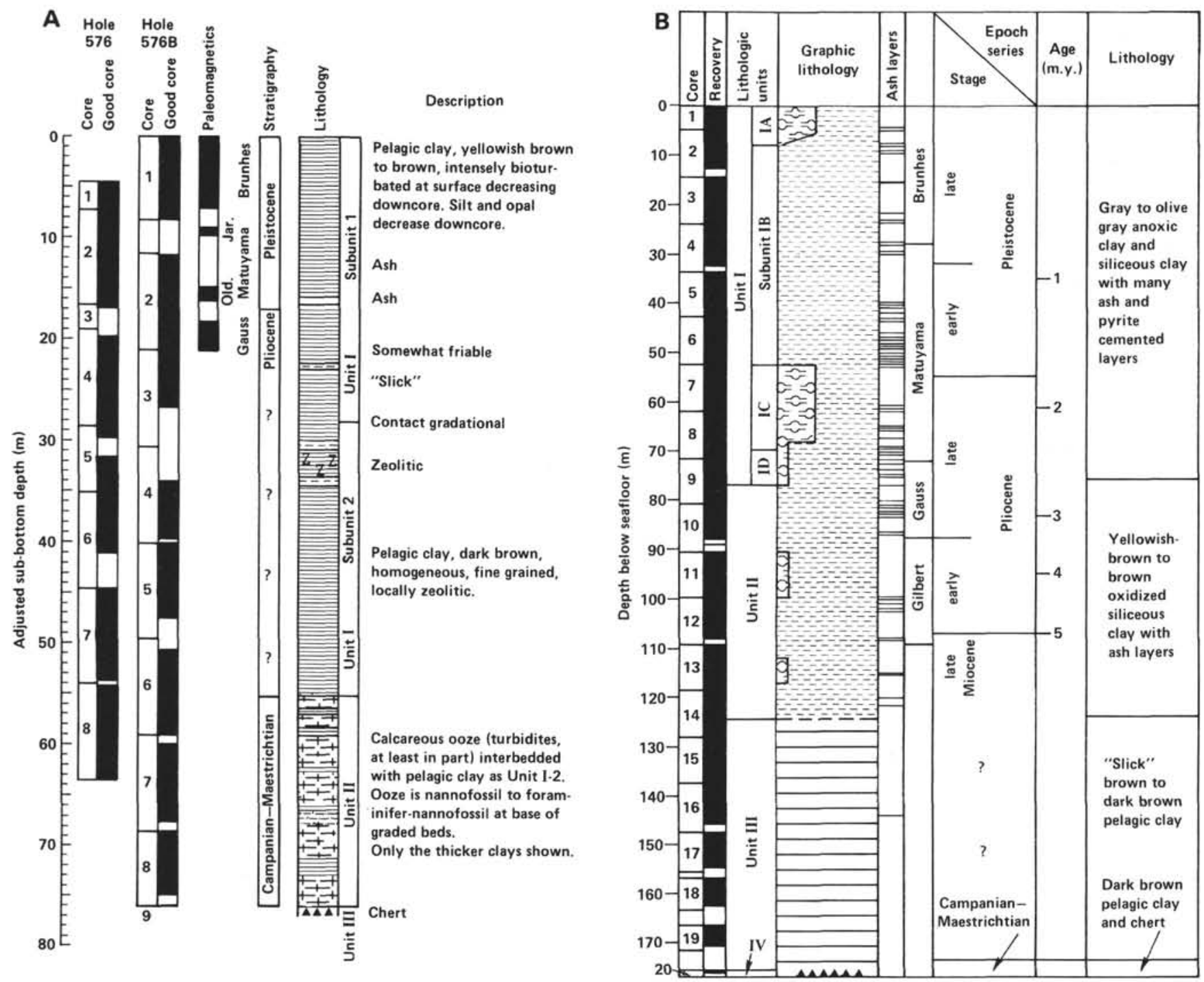

Figure 2. Lithologic columns constructed for (A) Site 576 and (B) Site 578. Paleomagnetic data from Heath, Rea, and Levi (this volume).

surface (4.77 m.y. ago to present). Below $25 \mathrm{~m}$ ichthyolith stratigraphic ranges constrain ages in the least-fossiliferous clays (Doyle and Riedel, this volume). Further age constraints are provided by foraminiferal and nannofossil datums in the carbonate oozes at the base of the hole (D'Agostino, this volume; Monechi, this volume). A smooth sedimentation-rate curve was constructed using the paleomagnetic data and microfossil age ranges (Fig. 3). Sedimentation rates were determined from the slope of the curve at 5-m.y. intervals and are included in Table 1. Through the upper $25 \mathrm{~m}$ the sedimentation rates decrease from $15 \mathrm{~m} / \mathrm{m}$.y. during the Brunhes Epoch to about $2 \mathrm{~m} / \mathrm{m}$.y. in the Gilbert Epoch. Sedimentation rates between 4.77 and $70 \mathrm{~m}$.y. ago range from $1 \mathrm{~m} / \mathrm{m}$.y. at $5 \mathrm{~m}$.y. ago to $0.3 \mathrm{~m} / \mathrm{m}$.y. at $35 \mathrm{~m}$.y. ago. Linear sedimentation rates increase slightly to about $0.6 \mathrm{~m} / \mathrm{m} . \mathrm{y}$. in the Late Cretaceous.

Paleomagnetic reversal stratigraphy (see Heath, Rea, and Levi, this volume) was used at Site 578 to calculate sedimentation rates. Sedimentation rates were calculated between the paleomagnetic reversal horizons (Fig. 3) and are included in Table 2. Sedimentation rates drop from $40 \mathrm{~m} / \mathrm{m}$.y. at the surface to about $25 \mathrm{~m} / \mathrm{m}$.y. around $2.0 \mathrm{~m}$.y. ago. Sedimentation rates decreased to less than $12 \mathrm{~m} / \mathrm{m} . \mathrm{y}$. around $3.0 \mathrm{~m} . \mathrm{y}$. ago and to about $8 \mathrm{~m} / \mathrm{m}$.y. 4.5 m.y. ago.

\section{Mass-Accumulation Rate Calculations}

To calculate the mass-accumulation rate of a sedimentary unit the actual dry mass of sediment per unit volume must be determined. The mass-accumulation rate is then the product of the linear sedimentation rate and this dry-bulk density. The dry-bulk density was determined by weighing, freeze-drying, and reweighing a known volume of original sample. Details of the procedures and calculations are found in Rea and Janecek (1981b). The calculated dry-bulk densities and mass-accumulation rates for Sites 576 and 578 are included in Tables 1 and 2, respectively. Eolian mass-accumulation rates were calculated by multiplying the eolian weight percentage by the total mass-accumulation rate and are presented in Tables 1 and 2 and Figures 4 and 5 . 
Table 1. Data generated for the calculation of eolian mass-accumulation rates and grain size at Site 576.

\begin{tabular}{|c|c|c|c|c|c|c|c|c|}
\hline $\begin{array}{c}\text { Sample } \\
\text { (interval in } \mathrm{cm} \text { ) }\end{array}$ & $\begin{array}{l}\text { Depth } \\
\text { (m) }\end{array}$ & $\begin{array}{c}\text { Age } \\
\text { (m.y.) }\end{array}$ & $\underset{(\mathrm{m} / \mathrm{m} . \mathrm{y} .)}{\text { LSR }}$ & $\underset{\left(\mathrm{gm} / \mathrm{cm}^{3}\right)}{\mathrm{DBD}}$ & $\begin{array}{c}\text { MAR } \\
\left(\mathrm{mg} / \mathrm{cm}^{2} 1000 \text { yr. }\right)\end{array}$ & $\begin{array}{l}\text { Eolian } \\
\text { wt. \% }\end{array}$ & $\begin{array}{c}\text { Eolian MAR } \\
\left(\mathrm{mg} / \mathrm{cm}^{2} 1000 \text { yr. }\right)\end{array}$ & $\begin{array}{c}\text { Eolian } \\
\text { size } \\
\left(\phi_{50}\right)\end{array}$ \\
\hline $1-6,30-33$ & 7.80 & 0.830 & 11.94 & 0.606 & 723 & 83.4 & 603 & 8.52 \\
\hline $2-1,90-93$ & 12.50 & 1.360 & 7.94 & 0.489 & 388 & 86.0 & 334 & 8.50 \\
\hline $2-2,90-93$ & 14.00 & 1.55 & 7.94 & 0.497 & 395 & 99.8 & 394 & 8.45 \\
\hline $2-3,90-93$ & 15.50 & 1.78 & 5.0 & 0.489 & 245 & 84.2 & 206 & 8.44 \\
\hline $3-5,20-23^{\mathrm{a}}$ & 15.80 & 1.84 & 5.0 & 0.521 & 261 & 79.3 & 207 & 8.34 \\
\hline $2-4,90-93$ & 17.00 & 2.12 & 4.07 & 0.575 & 234 & 88.0 & 206 & 8.37 \\
\hline $2-5,90-93$ & 18.50 & 2.50 & 3.11 & 0.553 & 172 & 87.7 & 151 & 8.43 \\
\hline $2-6,90-93$ & 20.00 & 2.96 & 4.28 & 0.569 & 244 & 93.8 & 229 & 8.35 \\
\hline $2-7,20-23$ & 20.80 & 3.21 & 3.18 & 0.616 & 200 & 84.4 & 169 & 8.37 \\
\hline $3-1,40-43$ & 21.50 & 3.43 & 2.71 & 0.593 & 212 & 86.8 & 184 & 8.52 \\
\hline $3-2,40-43$ & 23.00 & 3.99 & 3.08 & 0.614 & 189 & 87.9 & 166 & 8.54 \\
\hline $3-3,40-43$ & 24.50 & 4.66 & 2.00 & 0.694 & 139 & 87.1 & 121 & 8.60 \\
\hline $3-6,40-43$ & 24.89 & 4.80 & 1.00 & 0.689 & 69 & 85.8 & 59 & 8.59 \\
\hline $3-7,40-43$ & 26.39 & 6.3 & 1.00 & 0.671 & 67 & 81.6 & 55 & 8.58 \\
\hline $4-6,30-33^{a}$ & 26.90 & 6.8 & 1.00 & 0.651 & 65 & 82.0 & 53 & 8.60 \\
\hline $4-6,130-133^{\mathrm{a}}$ & 27.90 & 7.8 & 1.00 & 0.686 & 68 & 81.1 & 55 & 8.67 \\
\hline $5-1,40-43^{\mathrm{a}}$ & 28.60 & 9.3 & 0.6 & 0.670 & 40 & 79.8 & 32 & 8.73 \\
\hline $5-5,112-115^{\mathrm{a}}$ & 32.22 & 16.8 & 0.4 & 0.589 & 25 & 82.1 & 21 & 8.62 \\
\hline $5-6,30-33^{\mathrm{a}}$ & 32.90 & 18.5 & 0.4 & 0.681 & 27 & 87.1 & 24 & 8.61 \\
\hline $5-6,140-143^{\mathrm{a}}$ & 34.00 & 21.7 & 0.3 & 0.649 & 19 & 83.1 & 16 & 8.59 \\
\hline $5-7,20-23^{\mathrm{a}}$ & 34.30 & 22.7 & 0.3 & 0.540 & 16 & 85.1 & 14 & 8.54 \\
\hline $4-4,40-43$ & 35.00 & 25.0 & 0.3 & 0.579 & 17 & 81.6 & 14 & 8.59 \\
\hline $4-5,40-43$ & 36.50 & 29.0 & 0.3 & 0.601 & 18 & 79.5 & 14 & 8.78 \\
\hline $4-6,40-43$ & 38.00 & 34.5 & 0.3 & 0.606 & 18 & 79.8 & 15 & 8.93 \\
\hline $4-7,40-43$ & 39.50 & 36.7 & 0.4 & 0.660 & 20 & 84.7 & 17 & 8.98 \\
\hline $5-2,50-53$ & 40.60 & 40.0 & 0.4 & 0.592 & 18 & 88.2 & 16 & 8.94 \\
\hline $5-3,50-53$ & 42.10 & 43.8 & 0.4 & 0.588 & 23 & 90.3 & 21 & 8.80 \\
\hline $5-4,50-53$ & 43.60 & 47.5 & 0.4 & 0.579 & 37 & 99.9 & 37 & 8.90 \\
\hline $5-5,50-53$ & 45.10 & 51.5 & 0.45 & 0.780 & 35 & 98.7 & 35 & 8.41 \\
\hline $5-6,50-53$ & 46.60 & 55.00 & 0.5 & 0.775 & 39 & 99.9 & 39 & 8.42 \\
\hline $7-3,40-43^{\mathrm{a}}$ & 47.50 & 56.80 & 0.5 & 0.779 & 39 & 91.0 & 35 & 8.60 \\
\hline $7-4,40-43^{\mathrm{a}}$ & 49.00 & 59.80 & 0.5 & 0.790 & 40 & 89.7 & 35 & 8.60 \\
\hline $7-4,130-133^{\mathrm{a}}$ & 49.90 & 61.50 & 0.6 & 0.775 & 46 & 93.0 & 43 & 8.59 \\
\hline $6-1,130-133$ & 50.90 & 63.00 & 0.6 & 0.760 & 45 & 87.6 & 40 & 8.64 \\
\hline $6-2,130-133$ & 52.50 & 65.50 & 0.6 & 0.780 & 47 & 85.3 & 40 & 8.50 \\
\hline $6-3,130-133$ & 53.90 & 68.00 & 0.6 & 0.780 & 47 & 86.1 & 40 & 8.66 \\
\hline $6-4,50-53$ & 54.60 & 69.20 & 0.6 & 0.787 & 47 & 88.3 & 41 & 8.65 \\
\hline $6-5,110-113$ & 56.70 & 72.70 & 0.6 & 0.767 & 46 & 84.3 & 38 & 8.69 \\
\hline $6-6,75-78$ & 57.85 & 74.62 & 0.6 & 0.793 & 48 & 79.9 & 38 & 8.67 \\
\hline
\end{tabular}

Note: Depths are corrected according to the scheme outlined in the Site 576 chapter (this volume). LSR = linear sedimentation rate, DBD = dry-bulk density of the sample, MAR = mass-accumulation rate of the bulk sample. See text for an explanation of the calculations.

a Samples are from Hole 576. All other samples are from Hole 576B.

The precision of the chemical techniques was determined by replicate analyses and is less than $\pm 3 \%$. The accuracy of the final values for the mass-accumulation rates of the eolian component is determined largely by the accuracy of the stratigraphy. The placement of zonal boundaries, assuming constant linear sedimentation rates between boundaries and assignment of absolute ages to those boundaries almost certainly results in errors larger than the few percent that are the result of the laboratory work. Therefore, geological significance is not attached to changes in mass-accumulation rate of less than $10 \%$ of the values.

\section{RESULTS}

\section{Eolian Mass-Accumulation Rates}

In the Late Cretaceous, Paleocene, and early Eocene the eolian mass-accumulation rates at Site 576 (Fig. 4) remain relatively constant, averaging about $40 \mathrm{mg} / \mathrm{cm}^{2}$ / $10^{3} \mathrm{yr}$. Eolian accumulation rates then decline through the middle and late Eocene to about $15 \mathrm{mg} / \mathrm{cm}^{2} / 10^{3} \mathrm{yr}$., where they remain until the early Miocene. Eolian massaccumulation rates increase in the early Miocene to al- most $30 \mathrm{mg} / \mathrm{cm}^{2} / 10^{3} \mathrm{yr}$., then increase by more than a factor of two in the middle to late Miocene to $60 \mathrm{mg}$ / $\mathrm{cm}^{2} / 10^{3} \mathrm{yr}$. In the Pliocene, eolian accumulation rates increase dramatically to values over $400 \mathrm{mg} / \mathrm{cm}^{2} / 10^{3} \mathrm{yr}$.

Eolian accumulation rates at Site 578 (Fig. 5) are relatively low and constant during the interval from 4.5 to $3.0 \mathrm{~m}$.y. ago, averaging $500 \mathrm{mg} / \mathrm{cm}^{2} / 10^{3}$ yr. A peak in eolian accumulation rates at $2000 \mathrm{mg} / \mathrm{cm}^{2} / 10^{3} \mathrm{yr}$. occurs around 3.0 m.y. ago, followed by a permanent increase in eolian accumulation rates around $2.5 \mathrm{~m} . \mathrm{y}$. ago to values in the range of 1500 to $2500 \mathrm{mg} / \mathrm{cm}^{2} / 10^{3} \mathrm{yr}$. Samples younger than $2.5 \mathrm{~m}$.y. appear to exhibit greater variability, although this may be an artifact of the greater sample density in the upper portion of the core.

\section{Eolian Grain Size}

The size of the wind-borne grains at Site 576 increases from $8.70 \phi$ in the Late Cretaceous to $8.40 \phi$ in the Paleocene (Fig. 4), representing a $50 \%$ increase in grain mass. Eolian size decreases sharply to $8.85 \phi$ in the early Eocene and declines further to a low of $8.98 \phi$ in the earliest Oligocene, equivalent to an overall decrease in grain mass by a factor of 2.7. From this low in the early 
Table 2. Data generated for the calculation of eolian mass-accumulation rates and grain size at Site 578 .

\begin{tabular}{|c|c|c|c|c|c|c|c|c|}
\hline $\begin{array}{l}\text { Sample } \\
\text { (interval in } \mathrm{cm} \text { ) }\end{array}$ & $\begin{array}{l}\text { Depth } \\
\text { (m) }\end{array}$ & $\begin{array}{l}\text { Age } \\
(\mathrm{m} . \mathrm{y})\end{array}$ & $\begin{array}{c}\text { LSR } \\
(\mathrm{m} / \mathrm{m} . \mathrm{y} .)\end{array}$ & $\frac{\text { DBD }}{\left(\mathrm{gm} / \mathrm{cm}^{3}\right)}$ & $\begin{array}{c}\text { MAR } \\
\left(\mathrm{mg} / \mathrm{cm}^{2} 1000 \mathrm{yr}\right)\end{array}$ & $\begin{array}{l}\text { Eolian } \\
\text { wt. } \%\end{array}$ & $\begin{array}{c}\text { Eolian MAR } \\
\left(\mathrm{mg} / \mathrm{cm}^{2} 1000 \mathrm{yr} .\right)\end{array}$ & $\begin{array}{c}\text { Eolian } \\
\text { size } \\
\left(\phi_{50}\right)\end{array}$ \\
\hline $1-1,28-31$ & 0.28 & 0.007 & 37.4 & 0.583 & 2180 & 82.1 & 1790 & 7.89 \\
\hline $1-1,56-59$ & 0.56 & 0.015 & 37.4 & 0.517 & 1934 & 71.6 & 1385 & 7.94 \\
\hline $1-1,88-91$ & 0.88 & 0.024 & 37.4 & 0.520 & 1645 & 78.1 & 1519 & 8.12 \\
\hline $1-1,115-118$ & 1.15 & 0.031 & 37.4 & 0.541 & 2023 & 76.1 & 1539 & 8.40 \\
\hline $1-1,140-143$ & 1.40 & 0.037 & 37.4 & 0.512 & 1915 & 79.4 & 1521 & 8.13 \\
\hline $1-2,28-31$ & 1.78 & 0.048 & 37.4 & 0.580 & 2169 & 77.6 & 1683 & 8.30 \\
\hline $1-2,56-59$ & 2.06 & 0.055 & 37.4 & 0.593 & 2218 & 74.6 & 1655 & 7.93 \\
\hline $1-2,88-89$ & 2.38 & 0.064 & 37.4 & 0.525 & 1964 & 75.6 & 1485 & 8.07 \\
\hline $1-2,116-119$ & 2.66 & 0.071 & 37.4 & 0.546 & 2042 & 77.8 & 1589 & 8.05 \\
\hline $1-2,140-143$ & 3.00 & 0.080 & 37.4 & 0.553 & 2068 & 82.2 & 1699 & 8.06 \\
\hline $1-3,28-31$ & 3.28 & 0.088 & 37.4 & 0.520 & 1948 & 77.2 & 1504 & 8.30 \\
\hline $1-3,56-59$ & 3.56 & 0.096 & 37.4 & 0.616 & 2303 & 74.0 & 1704 & 7.85 \\
\hline $1-3,88-91$ & 3.88 & 0.104 & 37.4 & 0.723 & 2704 & 77.9 & 2106 & 7.94 \\
\hline $1-3,116-119$ & 4.16 & 0.111 & 37.4 & 0.671 & 2509 & 82.9 & 2080 & 8.00 \\
\hline $1-3,141-144$ & 4.41 & 0.118 & 37.4 & 0.619 & 2315 & 82.9 & 1919 & 8.10 \\
\hline $2-1,9-12$ & 4.89 & 0.131 & 37.4 & 0.559 & 2091 & 74.6 & 1559 & 8.16 \\
\hline $2-1,59-62$ & 5.39 & 0.144 & 37.4 & 0.666 & 2491 & 71.7 & 1786 & 8.14 \\
\hline $2-1,100-103$ & 5.80 & 0.155 & 37.4 & 0.538 & 2012 & 77.6 & 1561 & 7.94 \\
\hline $2-2,9-12$ & 6.39 & 0.171 & 37.4 & 0.593 & 2218 & 83.4 & 1850 & 7.93 \\
\hline $2-2,59-62$ & 6.89 & 0.184 & 37.4 & 0.556 & 2079 & 76.9 & 1599 & 7.85 \\
\hline $2-2,100-103$ & 7.30 & 0.195 & 37.4 & 0.632 & 2364 & 79.9 & 1888 & 8.16 \\
\hline $2-3,2-12$ & 7.89 & 0.211 & 37.4 & 0.601 & 2248 & 74.2 & 1668 & 8.30 \\
\hline $2-3,59-62$ & 8.39 & 0.225 & 37.4 & 0.588 & 2199 & 75.8 & 1667 & 7.66 \\
\hline $2-3,100-103$ & 8.80 & 0.236 & 37.4 & 0.634 & 2370 & 80.6 & 1910 & 7.75 \\
\hline $2-4,9-12$ & 9.39 & 0.251 & 37.4 & 0.697 & 2607 & 82.6 & 2153 & 7.59 \\
\hline $2-4,59-62$ & 9.89 & 0.265 & 37.4 & 0.723 & 2704 & 80.5 & 2176 & 7.60 \\
\hline $2-4,100-103$ & 10.30 & 0.276 & 37.4 & 0.707 & 2644 & 88.4 & 2337 & 7.64 \\
\hline $2-5,9-12$ & 10.89 & 0.291 & 37.4 & 0.606 & 2266 & 82.5 & 1869 & 8.24 \\
\hline $2-5,59-62$ & 11.39 & 0.305 & 37.4 & 0.640 & 2394 & 84.1 & 2013 & 7.74 \\
\hline $2-5,100-103$ & 11.80 & 0.316 & 37.4 & 0.624 & 2334 & 79.7 & 1860 & 7.80 \\
\hline $2-6,9-12$ & 12.39 & 0.332 & 37.4 & 0.572 & 2151 & 78.9 & 1697 & 7.77 \\
\hline $3-1,8-11$ & 14.38 & 0.385 & 37.4 & 0.694 & 2596 & 85.8 & 2227 & 8.14 \\
\hline $3-1,55-58$ & 14.85 & 0.397 & 37.4 & 0.654 & 2446 & 86.0 & 2103 & 8.07 \\
\hline $3-1,115-118$ & 15.45 & 0.414 & 37.4 & 0.627 & 2345 & 65.4 & 1534 & 7.86 \\
\hline $3-2,8-11$ & 15.88 & 0.425 & 37.4 & 0.615 & 2302 & 80.2 & 1846 & 7.90 \\
\hline $3-2,55-58$ & 16.35 & 0.438 & 37.4 & 0.606 & 2266 & 74.6 & 1690 & 8.02 \\
\hline $3-2,115-118$ & 16.95 & 0.454 & 37.4 & 0.598 & 2237 & 74.9 & 1675 & 8.11 \\
\hline $3-3,8-11$ & 17.38 & 0.465 & 37.4 & 0.627 & 2345 & 80.0 & 1876 & 8.21 \\
\hline $3-3,55-58$ & 17.85 & 0.478 & 37.4 & 0.608 & 2274 & 80.2 & 1824 & 7.63 \\
\hline $3-3,115-118$ & 18.45 & 0.494 & 37.4 & 0.627 & 2345 & 79.2 & 1857 & 7.60 \\
\hline $3-4,8-11$ & 18.88 & 0.505 & 37.4 & 0.671 & 2510 & 79.4 & 1993 & 8.35 \\
\hline $3-4,52-55$ & 19.32 & 0.517 & 37.4 & 0.653 & 2442 & 82.3 & 2009 & 8.02 \\
\hline $3-4,115-118$ & 19.95 & 0.534 & 37.4 & 0.666 & 2491 & 84.4 & 2102 & 7.65 \\
\hline $3-5,8-11$ & 20.38 & 0.545 & 37.4 & 0.733 & 2741 & 76.0 & 2083 & 7.53 \\
\hline $3-5,55-55$ & 20.82 & 0.557 & 37.4 & 0.675 & 2525 & 82.5 & 2084 & 7.97 \\
\hline $3-5,115-118$ & 21.45 & 0.575 & 37.4 & 0.640 & 2394 & 83.9 & 2002 & 7.66 \\
\hline $3-6,8-11$ & 21.88 & 0.586 & 37.4 & 0.790 & 2955 & 80.7 & 2385 & 8.14 \\
\hline $3-6,52-55$ & 22.32 & 0.597 & 37.4 & 0.762 & 2850 & 83.2 & 2371 & 8.08 \\
\hline $3-6,115-118$ & 22.95 & 0.614 & 37.4 & 0.655 & 2450 & 80.5 & 1972 & 7.77 \\
\hline $3-7,8-11$ & 23.38 & 0.626 & 37.4 & 0.624 & 2334 & 87.3 & 2037 & 7.70 \\
\hline $4-1,8-11$ & 23.88 & 0.639 & 37.4 & 0.832 & 3112 & 83.7 & 2604 & 7.70 \\
\hline $4-1,56-59$ & 24.36 & 0.652 & 37.4 & 0.732 & 2704 & 78.2 & 2115 & 7.99 \\
\hline $4-1,99-102$ & 24.79 & 0.644 & 37.4 & 0.806 & 3014 & 76.4 & 2302 & 8.32 \\
\hline $4-2,8-11$ & 25.38 & 0.678 & 37.4 & 0.647 & 3420 & 87.1 & 2108 & 7.98 \\
\hline $4-2,48-51$ & 25.78 & 0.690 & 37.4 & 0.798 & 2973 & 84.9 & 2525 & 7.94 \\
\hline $4-2,99-102$ & 26.29 & 0.704 & 37.4 & 0.694 & 2596 & 83.9 & 2178 & 7.55 \\
\hline $4-3,1-4$ & 26.81 & 0.718 & 37.4 & 0.718 & 2685 & 77.2 & 2072 & 7.90 \\
\hline $4-3,56-59$ & 27.36 & 0.736 & 28.8 & 0.694 & 2000 & 95.8 & 1916 & 7.70 \\
\hline $4-3,99-102$ & 27.79 & 0.751 & 28.8 & 0.655 & 1886 & 81.6 & 1543 & 8.27 \\
\hline $4-4,8-11$ & 28.38 & 0.771 & 28.8 & 0.671 & 1932 & 78.6 & 1519 & 7.80 \\
\hline $4-4,56-59$ & 28.86 & 0.788 & 28.8 & 0.679 & 1956 & 83.7 & 1637 & 7.73 \\
\hline $4-4,99-102$ & 29.29 & 0.803 & 28.8 & 0.757 & 2180 & 83.2 & 1813 & 7.86 \\
\hline $4-5,8-11$ & 29.88 & 0.823 & 28.8 & 0.751 & 2163 & 77.8 & 1683 & 8.00 \\
\hline $4-5,56-59$ & 30.36 & 0.840 & 28.8 & 0.668 & 1924 & 88.5 & 1703 & 8.04 \\
\hline $4-5,99-102$ & 30.79 & 0.855 & 28.8 & 0.619 & 1783 & 73.4 & 1309 & 8.05 \\
\hline $4-6,9-12$ & 31.39 & 0.876 & 28.8 & 0.829 & 2388 & 94.0 & 2245 & 7.64 \\
\hline $5-1,100-103$ & 34.30 & 0.936 & 54.0 & 0.624 & 3370 & 76.7 & 2584 & 8.09 \\
\hline $5-1,140-143$ & 34.70 & 0.947 & 29.0 & 0.744 & 2156 & 77.7 & 1677 & 8.07 \\
\hline $5-2,54-57$ & 35.34 & 0.969 & 29.0 & 0.707 & 2050 & 79.4 & 1628 & 8.15 \\
\hline $5-2,100-103$ & 35.80 & 0.985 & 29.0 & 0.707 & 2050 & 87.2 & 1789 & 8.01 \\
\hline $5-2,140-143$ & 36.20 & 0.999 & 29.0 & 0.679 & 1969 & 81.9 & 1613 & 7.86 \\
\hline $5-3,54-57$ & 36.84 & 1.021 & 29.0 & 0.655 & 1899 & 77.8 & 1477 & 8.13 \\
\hline $5-3,100-103$ & 37.30 & 1.037 & 29.0 & 0.621 & 1801 & 65.4 & 1178 & 7.94 \\
\hline $5-3,140-143$ & 37.70 & 1.050 & 29.0 & 0.673 & 1952 & 71.4 & 1394 & 7.93 \\
\hline $5-4,54-57$ & 38.34 & 1.073 & 29.0 & 0.707 & 2050 & 57.5 & 1179 & 8.16 \\
\hline $5-4,100-103$ & 38.80 & 1.088 & 29.0 & 0.749 & 2172 & 83.3 & 1809 & 7.80 \\
\hline $5-5,8-11$ & 39.38 & 1.108 & 29.0 & 0.679 & 1969 & 87.2 & 1717 & 7.85 \\
\hline
\end{tabular}


Table 2. (Continued).

\begin{tabular}{|c|c|c|c|c|c|c|c|c|}
\hline $\begin{array}{c}\text { Sample } \\
\text { (interval in } \mathrm{cm} \text { ) }\end{array}$ & $\begin{array}{l}\text { Depth } \\
\text { (m) }\end{array}$ & $\begin{array}{l}\text { Age } \\
(\mathrm{m} . \mathrm{y} .)\end{array}$ & $\begin{array}{c}\text { LSR } \\
(\mathrm{m} / \mathrm{m} . \mathrm{y} .)\end{array}$ & $\underset{\left(\mathrm{gm} / \mathrm{cm}^{3}\right)}{\text { DBD }}$ & $\begin{array}{c}\text { MAR } \\
\left(\mathrm{mg} / \mathrm{cm}^{2} 1000 \mathrm{yr} .\right)\end{array}$ & $\begin{array}{l}\text { Eolian } \\
\text { wt. \% }\end{array}$ & $\begin{array}{c}\text { Eolian MAR } \\
\left(\mathrm{mg} / \mathrm{cm}^{2} 1000 \mathrm{yr} .\right)\end{array}$ & $\begin{array}{c}\text { Eolian } \\
\text { size } \\
\left(\phi_{50}\right)\end{array}$ \\
\hline $5-5,60-63$ & 39.90 & 1.126 & 29.0 & 0.621 & 1801 & 89.0 & 1602 & 7.92 \\
\hline $5-5,100-103$ & 40.30 & 1.140 & 29.0 & 0.645 & 1871 & 85.5 & 1600 & 7.70 \\
\hline $5-6,33-36$ & 41.13 & 1.169 & 29.0 & 0.723 & 2097 & 91.9 & 1927 & 8.04 \\
\hline $5-6,100-103$ & 41.80 & 1.192 & 29.0 & 0.692 & 2007 & 91.3 & 1832 & 7.75 \\
\hline $5-7,33-36$ & 42.63 & 1.221 & 29.0 & 0.673 & 1952 & 86.6 & 1690 & 7.48 \\
\hline $6-1,57-60$ & 43.37 & 1.246 & 29.0 & 0.564 & 1636 & 83.4 & 1364 & 7.60 \\
\hline $6-1,100-103$ & 43.80 & 1.261 & 29.0 & 0.564 & 1636 & 86.1 & 1408 & 7.37 \\
\hline $6-1,140-143$ & 44.20 & 1.275 & 29.0 & 0.650 & 1885 & 81.3 & 1533 & 8.03 \\
\hline $6-2,52-55$ & 44.82 & 1.296 & 29.0 & 0.772 & 2239 & 82.9 & 1856 & 7.55 \\
\hline $6-2,100-103$ & 45.30 & 1.313 & 29.0 & 0.601 & 1743 & 77.4 & 1349 & 7.90 \\
\hline $6-2,140-143$ & 45.70 & 1.327 & 29.0 & 0.590 & 1711 & 81.7 & 1398 & 7.40 \\
\hline $6-3,30-33$ & 46.10 & 1.340 & 29.0 & 0.663 & 1923 & 89.1 & 1713 & 7.90 \\
\hline $6-3,95-98$ & 46.75 & 1.363 & 29.0 & 0.559 & 1621 & 81.6 & 1322 & 8.04 \\
\hline $6-4,15-18$ & 47.45 & 1.387 & 29.0 & 0.738 & 2140 & 86.7 & 1855 & 7.98 \\
\hline $6-4,90-93$ & 48.20 & 1.413 & 29.0 & 0.601 & 1743 & 81.7 & 1424 & 7.54 \\
\hline $6-4,140-143$ & 48.70 & 1.430 & 29.0 & 0.702 & 2036 & 84.0 & 1710 & 7.86 \\
\hline $6-5,50-53$ & 49.30 & 1.451 & 29.0 & 0.582 & 1688 & 99.0 & 1671 & 7.75 \\
\hline $6-5,90-93$ & 49.70 & 1.465 & 29.0 & 0.707 & 2050 & 90.3 & 1851 & 7.25 \\
\hline $6-5,140-143$ & 50.20 & 1.482 & 29.0 & 0.564 & 1636 & 79.9 & 1307 & 7.69 \\
\hline $6-6,47-50$ & 50.77 & 1.502 & 29.0 & 0.564 & 1636 & 82.8 & 1354 & 7.51 \\
\hline $6-6,100-103$ & 51.30 & 1.520 & 29.0 & 0.554 & 1607 & 81.7 & 1312 & 7.70 \\
\hline $6-7,37-40$ & 52.17 & 1.550 & 29.0 & 0.767 & 2224 & 85.9 & 1910 & 7.68 \\
\hline $7-1,12-15$ & 52.42 & 1.559 & 29.0 & 0.790 & 2291 & 80.5 & 1844 & 7.97 \\
\hline $7-1,60-63$ & 52.90 & 1.575 & 29.0 & 0.733 & 2126 & 74.1 & 1612 & 7.65 \\
\hline $7-1,110-113$ & 53.40 & 1.592 & 29.0 & 0.679 & 1969 & 87.8 & 1729 & 7.30 \\
\hline $7-2,10-13$ & 53.90 & 1.610 & 29.0 & 0.842 & 2442 & 82.6 & 2017 & 7.52 \\
\hline $7-2,60-63$ & 54.40 & 1.635 & 20.4 & 0.749 & 1535 & 82.6 & 1260 & 7.79 \\
\hline $7-2,135-138$ & 55.15 & 1.693 & 20.4 & 0.642 & 1316 & 75.0 & 987 & 7.74 \\
\hline $7-3,10-13$ & 55.40 & 1.711 & 20.4 & 0.655 & 1343 & 80.9 & 1086 & 7.43 \\
\hline $7-3,60-63$ & 55.90 & 1.750 & 20.4 & 0.702 & 1439 & 76.5 & 1100 & 7.67 \\
\hline $7-3,110-113$ & 56.40 & 1.788 & 20.4 & 0.975 & 1999 & 85.5 & 1709 & 7.41 \\
\hline $7-4,10-13$ & 56.90 & 1.826 & 20.4 & 0.772 & 1583 & 74.3 & 1176 & 7.80 \\
\hline $7-4,60-63$ & 57.40 & 1.864 & 20.4 & 0.603 & 1236 & 80.4 & 993 & 7.82 \\
\hline $7-4,110-113$ & 57.90 & 1.902 & 20.4 & 0.780 & 1599 & 95.7 & 1530 & 7.71 \\
\hline $7-5,10-13$ & 58.40 & 1.925 & 26.6 & 0.728 & 1936 & 83.4 & 1614 & 8.22 \\
\hline $7-5,60-63$ & 58.90 & 1.944 & 26.6 & 0.975 & 2594 & 85.0 & 2205 & 7.78 \\
\hline $7-5,110-113$ & 59.40 & 1.963 & 26.6 & 0.975 & 2594 & 82.7 & 2145 & 7.95 \\
\hline $7-6,10-13$ & 59.90 & 1.981 & 26.2 & 0.619 & 1647 & 79.4 & 1308 & 8.14 \\
\hline $7-6,60-63$ & 60.40 & 2.000 & 26.6 & 0.663 & 1764 & 81.8 & 1442 & 8.24 \\
\hline $7-6,110-113$ & 60.90 & 2.019 & 26.6 & 0.650 & 1729 & 82.9 & 1433 & 8.07 \\
\hline $7-7,12-15$ & 61.42 & 2.039 & 26.6 & 0.866 & 2304 & 91.8 & 2115 & 7.84 \\
\hline $8-1,20-23$ & 62.00 & 2.060 & 26.6 & 0.584 & 1553 & 81.0 & 1258 & 7.75 \\
\hline $8-1,74-77$ & 62.54 & 2.081 & 26.6 & 0.580 & 1543 & 84.4 & 1302 & 7.98 \\
\hline $8-1,120-123$ & 63.00 & 2.098 & 26.6 & 0.957 & 2546 & 88.5 & 2253 & 7.67 \\
\hline $8-2,20-23$ & 63.54 & 2.118 & 26.6 & 0.668 & 1777 & 77.1 & 1370 & 8.25 \\
\hline $8-2,74-77$ & 64.04 & 2.137 & 26.6 & 0.559 & 1487 & 83.9 & 1248 & 7.78 \\
\hline $8-2,120-123$ & 64.50 & 2.154 & 26.6 & 0.660 & 1756 & 82.5 & 1449 & 7.61 \\
\hline $8-3,20-23$ & 65.00 & 2.173 & 26.6 & 0.866 & 2304 & 86.0 & 1981 & 7.49 \\
\hline $8-3,74-77$ & 65.54 & 2.193 & 26.6 & 0.718 & 1910 & 83.7 & 1599 & 7.77 \\
\hline $8-3,120-123$ & 66.00 & 2.211 & 26.6 & 0.686 & 1826 & 95.0 & 1734 & 7.80 \\
\hline $8-4,20-23$ & 66.50 & 2.229 & 26.6 & 0.647 & 1721 & 87.2 & 1500 & 7.45 \\
\hline $8-4,75-78$ & 67.06 & 2.250 & 26.6 & 0.611 & 1625 & 79.2 & 1287 & 7.48 \\
\hline $8-4,130-133$ & 67.60 & 2.271 & 26.6 & 0.646 & 1718 & 83.5 & 1434 & 8.20 \\
\hline $8-5,20-23$ & 68.00 & 2.286 & 26.6 & 0.671 & 1785 & 76.4 & 1364 & 7.83 \\
\hline $8-5,74-77$ & 68.54 & 2.306 & 26.6 & 0.842 & 2240 & 81.3 & 1821 & 8.22 \\
\hline $8-5,123-128$ & 69.05 & 2.325 & 26.6 & 0.985 & 2620 & 83.3 & 2183 & 7.53 \\
\hline $8-6,20-23$ & 69.50 & 2.342 & 26.6 & 1.118 & 2974 & 79.4 & 2361 & 8.13 \\
\hline $8-6,60-63$ & 69.90 & 2.357 & 26.6 & 0.772 & 2054 & 84.5 & 1736 & 8.19 \\
\hline $8-6,114-117$ & 70.44 & 2.378 & 26.6 & 0.803 & 2136 & 83.7 & 1788 & 8.26 \\
\hline $8-7,24-27$ & 71.04 & 2.400 & 26.6 & 0.816 & 2171 & 88.4 & 1919 & 8.20 \\
\hline $9-1,17-20$ & 71.47 & 2.416 & 26.6 & 0.842 & 2240 & 79.9 & 1789 & 7.86 \\
\hline $9-1,67-70$ & 71.97 & 2.435 & 26.6 & 0.881 & 2343 & 81.4 & 1907 & 7.95 \\
\hline $9-1,114-117$ & 72.44 & 2.453 & 26.6 & 0.723 & 1923 & 81.7 & 1571 & 8.06 \\
\hline $9-2,17-20$ & 72.97 & 2.474 & 17.0 & 1.069 & 1817 & 88.4 & 1606 & 8.05 \\
\hline $9-2,71-74$ & 73.51 & 2.506 & 17.0 & 0.780 & 1326 & 77.1 & 1022 & 7.83 \\
\hline $9-2,114-117$ & 73.95 & 2.531 & 17.0 & 0.774 & 1265 & 82.1 & 1038 & 8.45 \\
\hline $9-3,17-20$ & 74.47 & 2.562 & 17.0 & 0.889 & 1511 & 84.0 & 1269 & 7.83 \\
\hline $9-3,67-70$ & 74.97 & 2.591 & 17.0 & 0.629 & 1069 & 81.2 & 868 & 8.20 \\
\hline $9-3,114-117$ & 75.44 & 2.619 & 17.0 & 0.655 & 1114 & 81.0 & 902 & 8.03 \\
\hline $9-4,17-20$ & 75.97 & 2.650 & 17.0 & 0.842 & 1431 & 72.0 & 1030 & 8.07 \\
\hline $9-4,47-50$ & 76.27 & 2.668 & 17.0 & 0.897 & 1525 & 89.0 & 1369 & 8.23 \\
\hline $9-4,114-117$ & 76.95 & 2.707 & 17.0 & 0.650 & 1105 & 78.3 & 865 & 8.29 \\
\hline $9-5,30-33$ & 77.63 & 2.747 & 17.0 & 0.634 & 1078 & 78.7 & 848 & 7.95 \\
\hline $9-5,67-70$ & 77.97 & 2.767 & 17.0 & 0.642 & 1091 & 78.7 & 859 & 8.22 \\
\hline $9-5,105-108$ & 78.35 & 2.790 & 17.0 & 0.874 & 1486 & 72.8 & 1082 & 8.05 \\
\hline $9-6,14-17$ & 78.94 & 2.824 & 17.0 & 0.874 & 1486 & 85.9 & 1277 & 8.31 \\
\hline $9-6,54-57$ & 79.34 & 2.848 & 17.0 & 0.702 & 1193 & 76.6 & 914 & 8.20 \\
\hline
\end{tabular}


Table 2. (Continued).

\begin{tabular}{|c|c|c|c|c|c|c|c|c|}
\hline $\begin{array}{c}\text { Sample } \\
\text { (interval in } \mathrm{cm} \text { ) }\end{array}$ & $\begin{array}{l}\text { Depth } \\
\text { (m) }\end{array}$ & $\begin{array}{l}\text { Age } \\
\text { (m.y.) }\end{array}$ & $\underset{(\mathrm{m} / \mathrm{m} \cdot \mathrm{y} .)}{\operatorname{LSR}}$ & $\underset{\left(\mathrm{gm} / \mathrm{cm}^{3}\right)}{\mathrm{DBD}}$ & $\begin{array}{c}\text { MAR } \\
\text { (mg/cm² } 1000 \mathrm{yr} .)\end{array}$ & $\begin{array}{l}\text { Eolian } \\
\text { wt. \% }\end{array}$ & $\begin{array}{c}\text { Eolian MAR } \\
\left(\mathrm{mg} / \mathrm{cm}^{2} 1000 \text { yr. }\right)\end{array}$ & $\begin{array}{c}\text { Eolian } \\
\text { size } \\
\left(\phi_{50}\right)\end{array}$ \\
\hline $9-6,97-100$ & 79.77 & 2.873 & 17.0 & 0.794 & 1351 & 83.3 & 1113 & 7.86 \\
\hline $9-7,14-17$ & 80.44 & 2.912 & 22.2 & 0.939 & 2085 & 82.1 & 1712 & 7.90 \\
\hline $10-1,9-12$ & 80.89 & 2.932 & 22.2 & 1.048 & 2327 & 80.4 & 1871 & 7.72 \\
\hline $10-1,56-59$ & 81.36 & 2.953 & 22.2 & 1.009 & 2220 & 81.3 & 1805 & 8.20 \\
\hline $10-1,108-111$ & 81.88 & 2.977 & 22.2 & 0.975 & 2165 & 73.8 & 1598 & 8.37 \\
\hline $10-2,9-12$ & 82.39 & 3.000 & 17.1 & 0.858 & 1461 & 81.8 & 1195 & 8.04 \\
\hline $10-2,62-65$ & 82.92 & 3.030 & 17.1 & 0.744 & 1272 & 83.8 & 1066 & 8.20 \\
\hline $10-2,108-111$ & 83.38 & 3.057 & 17.1 & 0.647 & 1106 & 82.4 & 911 & 8.16 \\
\hline $10-3,9-12$ & 83.89 & 3.091 & 14.0 & 0.702 & 983 & 80.0 & 786 & 8.30 \\
\hline $10-3,56-59$ & 84.36 & 3.124 & 14.0 & 0.718 & 1005 & 84.1 & 845 & 8.53 \\
\hline $10-3,108-111$ & 84.88 & 3.161 & 14.0 & 0.728 & 1019 & 87.0 & 886 & 8.42 \\
\hline $10-4,9-12$ & 85.30 & 3.197 & 11.3 & 0.728 & 823 & 79.4 & 653 & 8.53 \\
\hline $10-4,56-59$ & 85.86 & 3.246 & 11.3 & 0.738 & 834 & 81.2 & 677 & 7.95 \\
\hline $10-4,102-105$ & 86.32 & 3.287 & 11.3 & 0.650 & 735 & 83.2 & 611 & 8.46 \\
\hline $10-5,9-12$ & 86.89 & 3.338 & 11.3 & 0.827 & 935 & 75.6 & 707 & 8.36 \\
\hline $10-5,56-59$ & 87.36 & 3.380 & 11.3 & 0.640 & 723 & 79.5 & 575 & 8.40 \\
\hline $10-5,108-111$ & 87.88 & 3.423 & 13.9 & 0.718 & 998 & 82.6 & 824 & 8.05 \\
\hline $10-6,9-12$ & 88.39 & 3.460 & 13.9 & 0.874 & 1215 & 78.2 & 950 & 8.03 \\
\hline $10-6,56-59$ & 88.86 & 3.493 & 13.9 & 0.660 & 917 & 85.3 & 754 & 8.44 \\
\hline $11-1,40-43$ & 90.70 & 3.626 & 13.9 & 0.686 & 954 & 75.0 & 715 & 8.00 \\
\hline $11-1,80-83$ & 91.10 & 3.655 & 13.9 & 0.670 & 931 & 76.8 & 715 & 8.14 \\
\hline $11-1,134-137$ & 91.64 & 3.693 & 13.9 & 0.640 & 890 & 77.2 & 687 & 8.09 \\
\hline $11-2,40-42$ & 92.20 & 3.734 & 13.9 & 0.661 & 919 & 75.1 & 690 & 8.19 \\
\hline $11-2,80-83$ & 92.60 & 3.762 & 13.9 & 0.772 & 1073 & 93.3 & 1001 & 8.01 \\
\hline $11-2,138-141$ & 93.18 & 3.804 & 13.9 & 0.881 & 1225 & 79.9 & 979 & 8.20 \\
\hline $11-3,30-33$ & 93.60 & 3.835 & 13.0 & 0.707 & 919 & 78.2 & 719 & 8.20 \\
\hline $11-3,77-80$ & 94.07 & 3.872 & 13.0 & 0.749 & 974 & 75.3 & 733 & 8.25 \\
\hline $11-3,122-125$ & 94.52 & 3.906 & 13.0 & 0.738 & 959 & 74.5 & 714 & 8.30 \\
\hline $11-4,40-43$ & 95.20 & 3.964 & 11.3 & 0.837 & 946 & 85.5 & 809 & 8.13 \\
\hline $11-4,80-83$ & 95.60 & 3.999 & 11.3 & 0.697 & 788 & 81.8 & 645 & 8.17 \\
\hline $11-4,122-125$ & 96.02 & 4.036 & 11.3 & 0.707 & 799 & 81.0 & 647 & 8.37 \\
\hline $11-5,40-43$ & 96.70 & 4.112 & 7.2 & 0.733 & 528 & 82.7 & 436 & 8.25 \\
\hline $11-5,77-80$ & 97.07 & 4.163 & 7.2 & 0.749 & 539 & 85.4 & 460 & 8.43 \\
\hline $11-5,122-125$ & 97.52 & 4.225 & 7.4 & 0.785 & 565 & 86.5 & 489 & 7.90 \\
\hline $11-6,41-44$ & 98.21 & 4.319 & 7.4 & 0.692 & 512 & 85.0 & 435 & 8.35 \\
\hline $11-6,122-125$ & 99.02 & 4.429 & 7.4 & 0.767 & 568 & 79.8 & 453 & 8.33 \\
\hline
\end{tabular}

Note: Abbreviations are as in Table 1.

Oligocene, the size of the eolian material steadily increases to $8.55 \phi$ in the early Miocene. Following a decrease in size to $8.73 \phi$ in the middle Miocene, eolian size increases through the late Miocene and Pliocene to $8.35 \phi$. The increases in size during the Oligocene and late Miocene/Pliocene are equivalent to changes in grain mass by a factor of 2.4 and 2.1 , respectively.

Between 4.5 and 2.5 m.y. ago the average eolian grain size at Site 578 (Fig. 5) was relatively small, generally less than $8.00 \phi$. The size of the eolian material increases from an average of $8.20 \phi$ at $2.5 \mathrm{~m} . \mathrm{y}$. ago to an average of $7.7 \phi$ at $1.5 \mathrm{~m} . \mathrm{y}$. ago, then decreases to an average of $8.00 \phi$ over the last million years.

\section{DISCUSSION}

\section{Comparison of DSDP Site 576 and LL44-GPC-3}

The record of eolian accumulation and grain size at DSDP Site 576 provides an excellent complement to the record at LL44-GPC-3 (Leinen and Heath, 1981, Janecek and Rea, 1983; Leinen, this volume), located $3500 \mathrm{~km}$ to the east at approximately the same latitude (Fig. 1). The eolian mass-accumulation rate and the grain size for both sites are plotted in Figure 6.

During the Late Cretaceous and Paleocene, Site 576 was moving northward on the Pacific plate under the northeast trade winds (Fig. 7). Eolian accumulation rates were relatively low, averaging about $40 \mathrm{mg} / \mathrm{cm}^{2} / 10^{3} \mathrm{yr}$. Low eolian input is in agreement with independent data suggesting warmer (Savin, 1977; Frakes, 1979) and wetter (Wolfe and Hopkins, 1967; Wolfe, 1978; Kemp, 1978) climatic conditions during the early Cenozoic than at the present. In addition, the lack of glacial erosion products would mean less material was available for erosion and transportation. Eolian accumulation rates decreased further to less than $20 \mathrm{mg} / \mathrm{cm}^{2} / 10^{3}$ yr. during the middle and late Eocene and may be indicative of the humid climates that characterized that time (cf. Frakes, 1979) and/or the passage of the site out of the core of the trade winds (Leinen and Heath, 1981). The low accumulation rates in the Oligocene contrast with other climatic data (cf. Frakes, 1979) that suggest drier conditions in the Oligocene than in the Paleocene and Eocene.

The eolian accumulation records at Site 576 and LL44GPC-3 are very similar for the Late Cretaceous and early Cenozoic; both are characterized by relatively low rates. However, eolian accumulation rates at Site 576 are two to three times greater than at LL44-GPC-3. In addition, the record at LL44-GPC-3 is marked by an accumulation rate maximum in the early Eocene that is not present at Site 576 (Fig. 6).

The early Eocene accumulation maximum at LL44GPC-3 has been interpreted by Leinen and Heath (1981) to be the result of the site moving through the zone of 

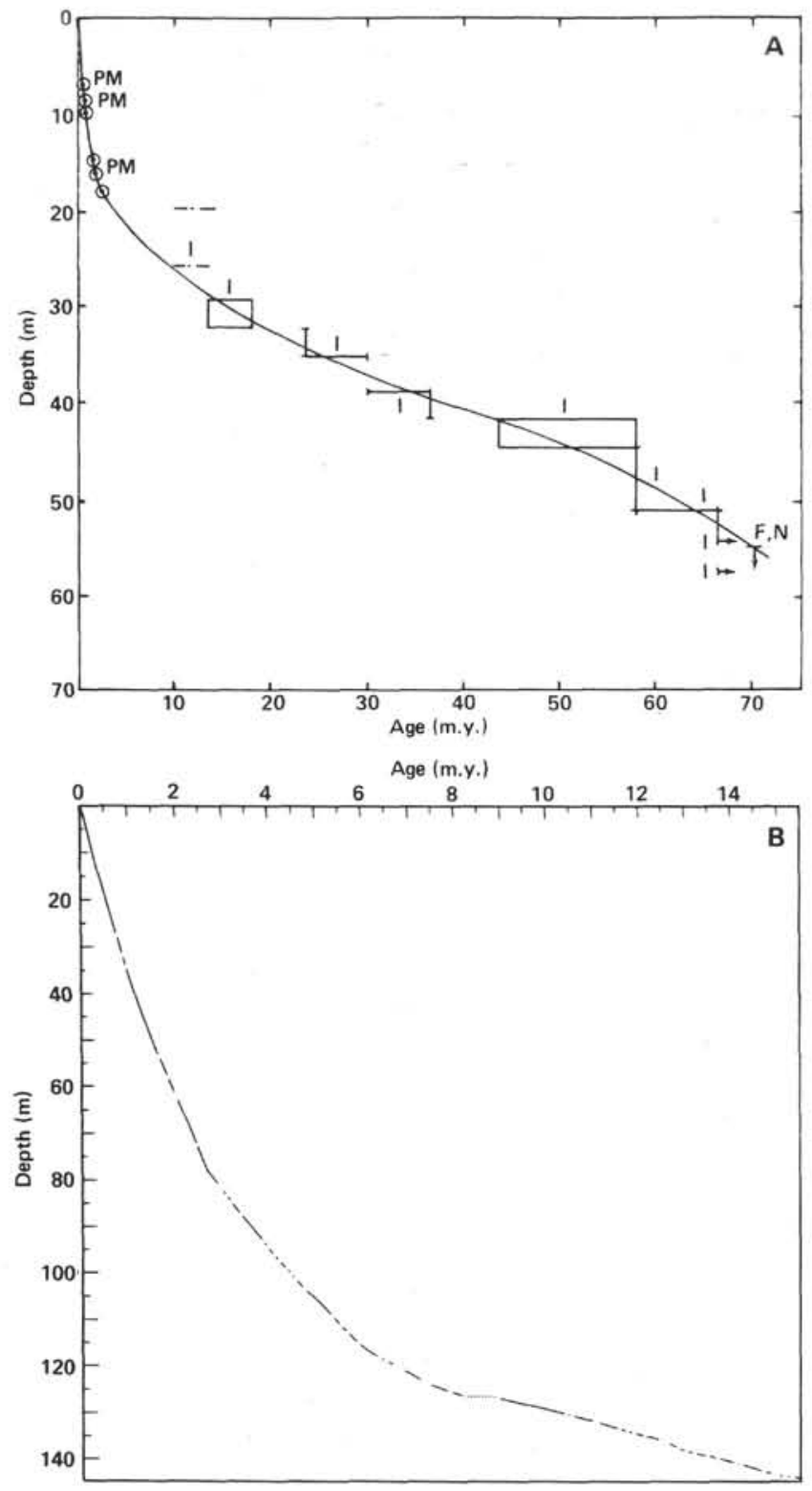

Figure 3. Depth versus age plot. A. Site 576. B. Site 578. Paleomagnetic (PM) data from Heath, Rea, and Levi (this volume). Foraminiferal (F) age ranges from D'Agostino (this volume). Nannofossil (N) age ranges from Monechi (this volume). Ichthyolith (I) age ranges from Doyle and Riedel (this volume).

the maximum northeast trade wind influence in the eastern Pacific. The lack of similar pattern at Site $\mathbf{5 7 6}$ may be the result of poorly constrained biostratigraphy.

The higher early Cenozoic eolian accumulation rates at Site 576 relative to LL44-GPC-3 are interesting in that LL44-GPC-3 is $3500 \mathrm{~km}$ closer to the predominant source regions in Central America and Mexico and might be expected to have greater eolian accumulation rates. The difference, again, may be the result of poorly constrained biostratigraphy at both sites. Another possible explanation, however, is that Site 576 was receiving significant input from Asia as well as from Central America. Duce et al. (1980) have shown that Asian dust can contribute significantly to the total marine aerosol at lo- cations in the trade winds. At present, dust originating in Asia is carried eastward by the high-level westeriies, injected below the trade-wind inversion by subsidence associated with anticyclones moving off of Japan in the spring of the year, and carried westward by the trade winds. If such trajectories occurred in the early Cenozoic, Site 576 would have been influenced more by these circulation patterns than LL44-GPC-3 and, thus, would record higher eolian input.

Distinct changes in eolian accumulation rates and in the mineralogy of bulk sediment (Leinen, this volume; Schoonmaker et al., this volume) at $25 \mathrm{~m}$.y. ago may be the result of the two sites moving into the influence of the westerlies at this time. Schoonmaker et al. (this volume) record an increase in quartz and feldspar content as well as an increase in mixed layer illite to smectite ratio at Site 576 around $20-25$ m.y. ago. Leinen (this volume) shows an increase in quartz content at $28 \mathrm{~m}$.y. ago and a gradual increase between 18 and 8 m.y. ago at Site 576. Leinen and Heath (1981) recorded a large change in the quartz content and chemistry of the sediments at LL44-GPC-3 approximately 23 m.y. ago. In addition, they report a gradual increase in quartz content between 18 and $8 \mathrm{~m}$.y. ago. The temporal difference in the initial increase in quartz content at Site 576 is probably the result of the greater sample density used by Leinen in her study. Thus, the mineralogy data suggest a change in source material from quartz-poor, more basic dust before the Miocene to a more quartz-rich material from the Asian continent after the sites moved into the influence of the westerlies between 20 and 28 m.y. ago (Leinen and Heath, 1981). The details of that transition time remain unclear, however, because of stratigraphic uncertainty.

Eolian accumulation rates underwent another increase at both sites between 15 and $10 \mathrm{~m} . \mathrm{y}$. ago (Fig. 6), a time coincident with the onset of rapid ice-volume increase in Antarctica (Savin, 1977; Brewster, 1980; Woodruff et al., 1981). This increase in the mid-to-late Miocene, along with a factor of six increase in accumulation rates in Pliocene/Pleistocene time, supports the general correlation between glacial ages, global aridity, and an increase in glacial erosion products for dust transport (Rea and Janecek, 1982).

\section{Eolian Grain Size and Atmosphere Circulation}

The size of wind-borne grains at Site 576 (Fig. 4) increased from the Late Cretaceous into the Paleocene, suggesting a slight increase in the intensity of atmospheric circulation across the Cretaceous/Tertiary boundary. An increase in quartz grain size (Janecek and Rea, 1983) is also seen at LL44-GPC-3 over this same time interval, although the trend in the total eolian component is less obvious (Fig. 6). Thus, there may not be a significant change in atmospheric circulation across the Cretaceous/ Tertiary boundary. The sampling interval in these cores, however, was not designed to resolve short-duration phenomena.

The eolian grain size at Site 576 in the Late Cretaceous and Paleocene is equivalent to that in the Pliocene and Pleistocene, two very different climatic intervals. 


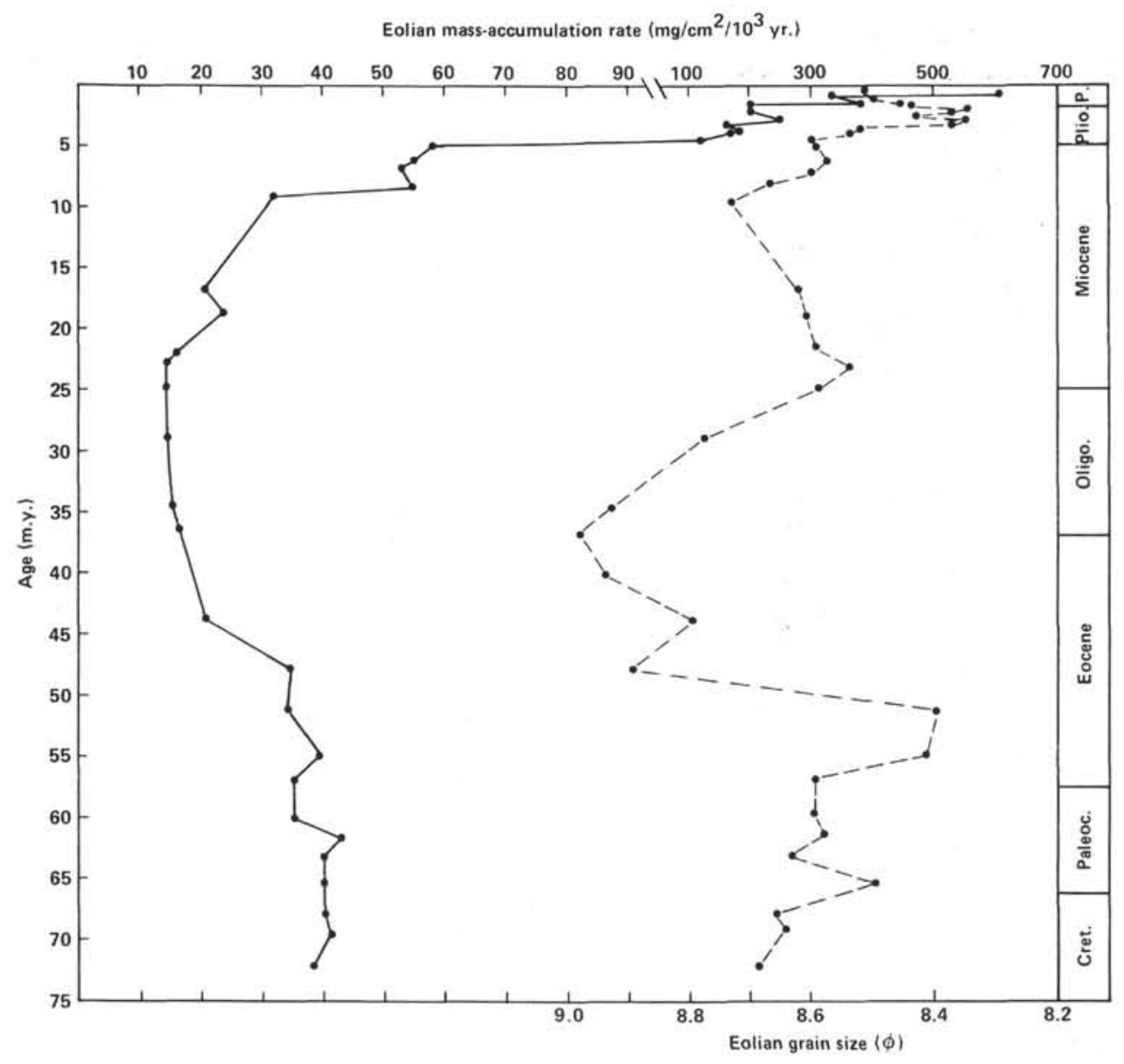

Figure 4. Eolian mass-accumulation rates (solid line) and eolian grain size (dashed line) for Site 576.

Global atmospheric circulation models developed by Barron and Washington (1982) that incorporate Cretaceous paleogeography suggest that Cretaceous wind speeds may have been increased relative to the present and were much greater than would be inferred from Cretaceous pole-toequator temperature gradients. The eolian size data from Site 576 and LL44-GPC- 3 are in agreement with relatively high Cretaceous wind intensities.

A sharp reduction in the eolian grain size at Site 576 near the Paleocene/Eocene boundary could represent a significant reduction in the intensity of atmospheric circulation. Over this relatively short time span, the eolian grain mass is reduced by a factor of three at Site 576; a change on the order of the one that occurs between the glacial-to-interglacial oscillations in the late Pleistocene (Janecek and Rea, in press). A similar, although larger, size decrease occurs at LL44-GPC-3 (Fig. 6) at this time (Janecek and Rea, 1983). Diagenetic effects resulting in increased grain size in the older sediments are not the cause of the size change, since scanning electron microscopy of the eolian material at LL44-GPC-3 (Janecek and Rea, 1983) and at Site 576 shows no grain overgrowths or cementation in the older sediments. Since the eolian material is in equilibrium with the transporting wind because of the large transport distances involved, the introduction of material from a more distant source area should have little, if any, effect on the size of the eolian grains. This sharp decrease in grain size might be associated with a reduction in pole-to-equator temperature gradients at this time, although there is little other independent paleoclimatic information to suggest such a large change in climate in the early Eocene.

The relatively low grain-size values in the Eocene and early Oligocene imply that the vigor of atmospheric circulation was lower at that time than at any other time in the Cenozoic. This scenario is supported by planktonic and benthic ${ }^{18} \mathrm{O}$ data (Savin, 1977), which indicate low latitudinal thermal gradients and hence sluggish atmospheric circulation during the Eocene and early Oligocene. Following the low in eolian grain size in the late Eocene and early Oligocene, eolian grain size at both sites increased steadily into the early Miocene. The increase in wind intensity suggested by this information corresponds well with the sudden onset of polar cooling and cold bottom-water formation about 38 m.y. ago (Kennett and Shackleton, 1976).

The decrease in grain size at both sites in the early Miocene (Fig. 6) may be the result of a slight warming trend at that time. Oxygen isotopes (Woodruff et al., 1981 ) indicate an increase in temperature in the early Miocene and thus, possibly a reduction in pole-to-equator temperature gradients and in the intensity of the zonal winds. The reduction in grain size also may be, in part, the result of the light and variable winds associated with 


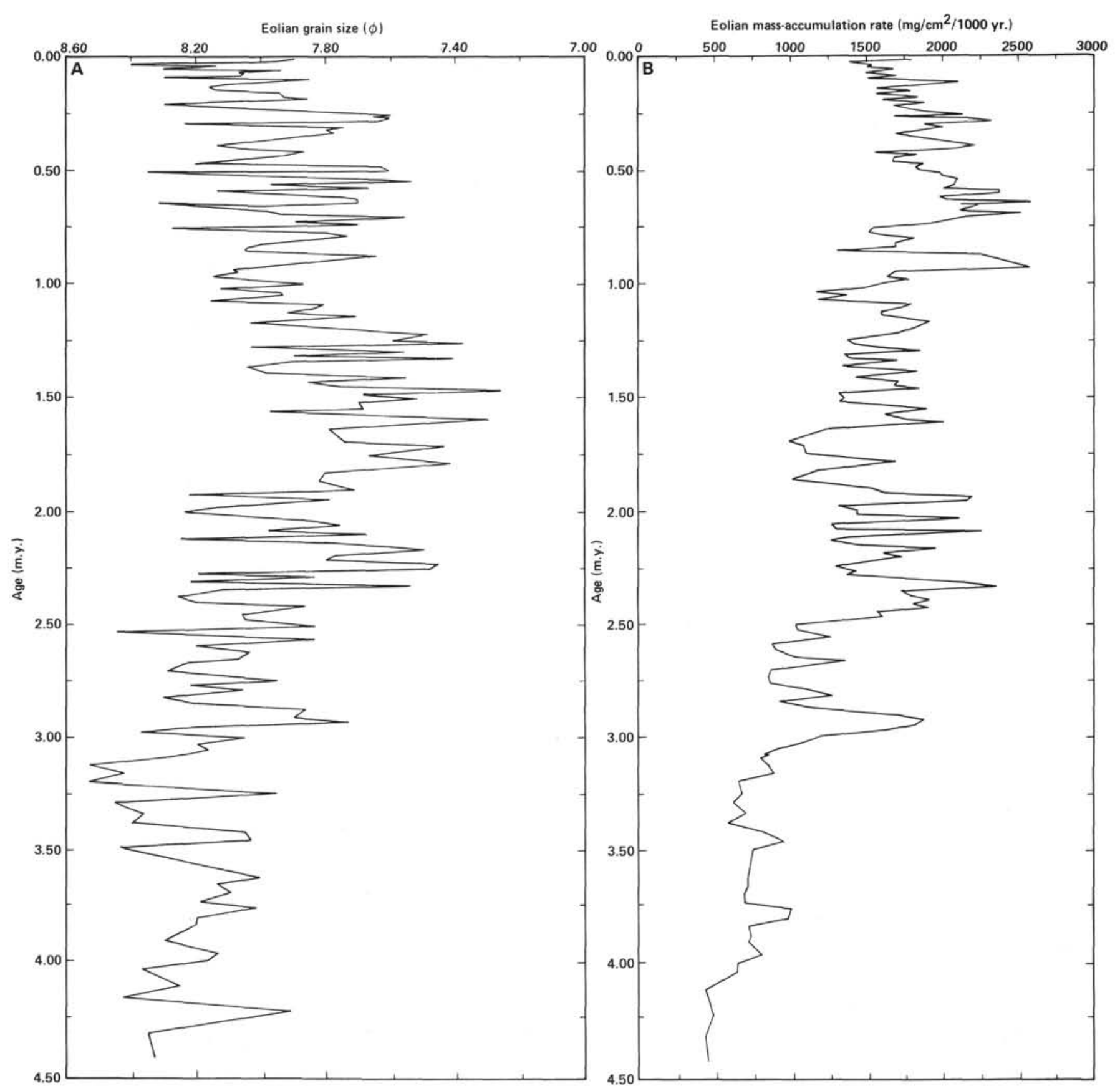

Figure 5. (A) Eolian grain size and (B) eolian mass-accumulation rates for Site 578.

the transition zone between the prevailing westerlies and the trade winds.

The size of the wind-borne material at LL44-GPC-3 (Fig. 6) increased during the Miocene, suggesting an intensification of atmospheric circulation, apparently the result of polar cooling (Bandy et al., 1969; Savin, 1977; Woodruff et al., 1981). However, the record at Site 576 shows an overall decrease in grain size between 10 and 20 m.y. ago. Because the record at Site 576 over this time interval is constrained by only three points, it is unclear to what degree the data actually conflict. Both sites exhibit generally coarsening grain-size trends in samples younger than $10 \mathrm{~m} . \mathrm{y}$. A relatively smaller increase in grain size in the Pliocene than in the Miocene and Oligocene suggests that larger increases in atmospheric circulation occurred during the Oligocene and possibly the Miocene than during the onset of Northern Hemisphere glaciation.

\section{The Onset of Northern Hemisphere Glaciation}

The eolian accumulation rate and grain-size data for Site 576 and LL44-GPC-3 increase dramatically in the mid-Pliocene, coincident with the onset of Northern Hemisphere glaciation. However, the low sedimentation rates in these cores make it difficult to establish the timing of the changes in eolian deposition with the onset of North- 

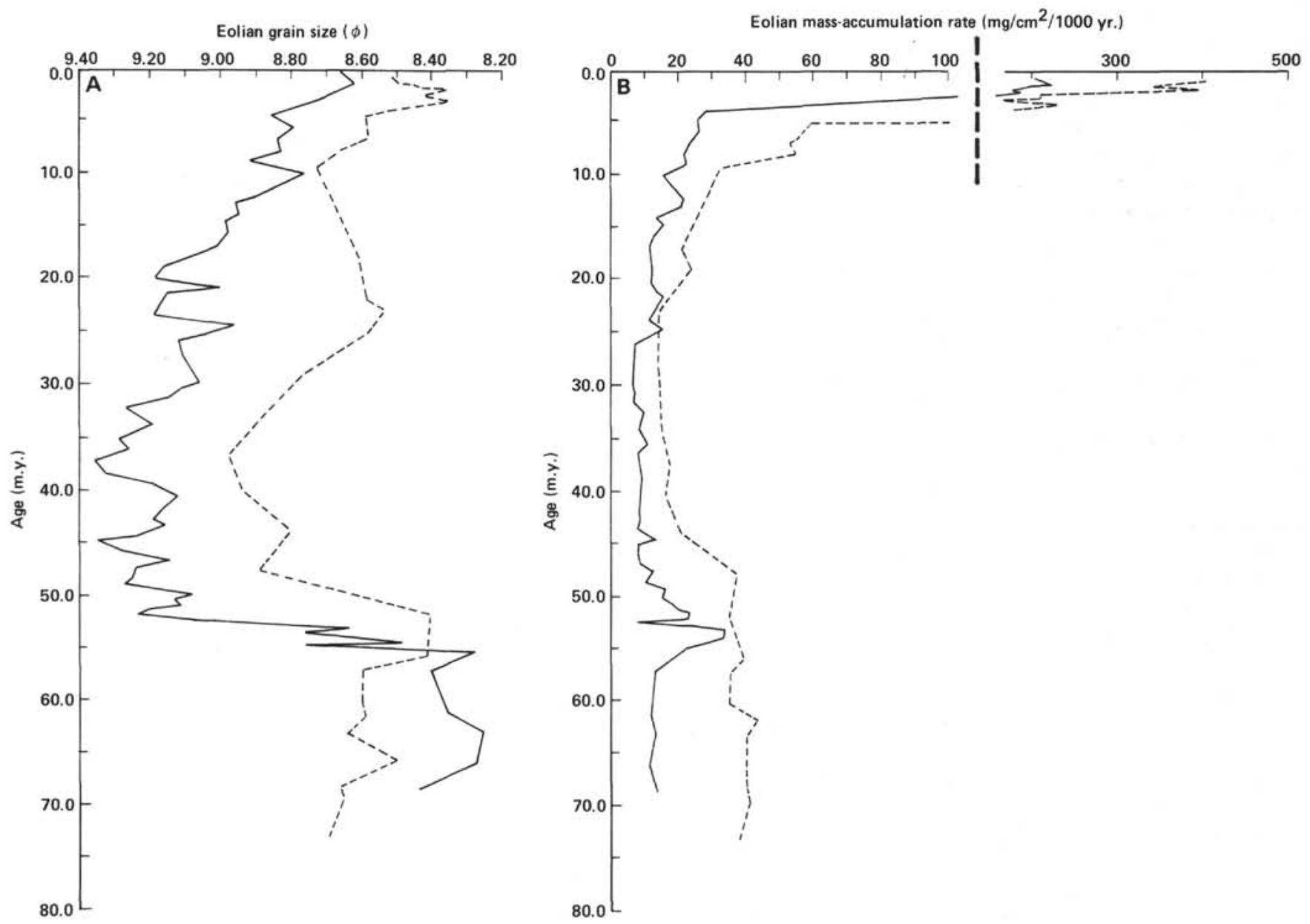

Figure 6. (A) Site 576 (dashed line) eolian grain size and (B) mass-accumulation rates plotted with respective components from LL44-GPC-3 (solid line) (Janecek and Rea 1983).

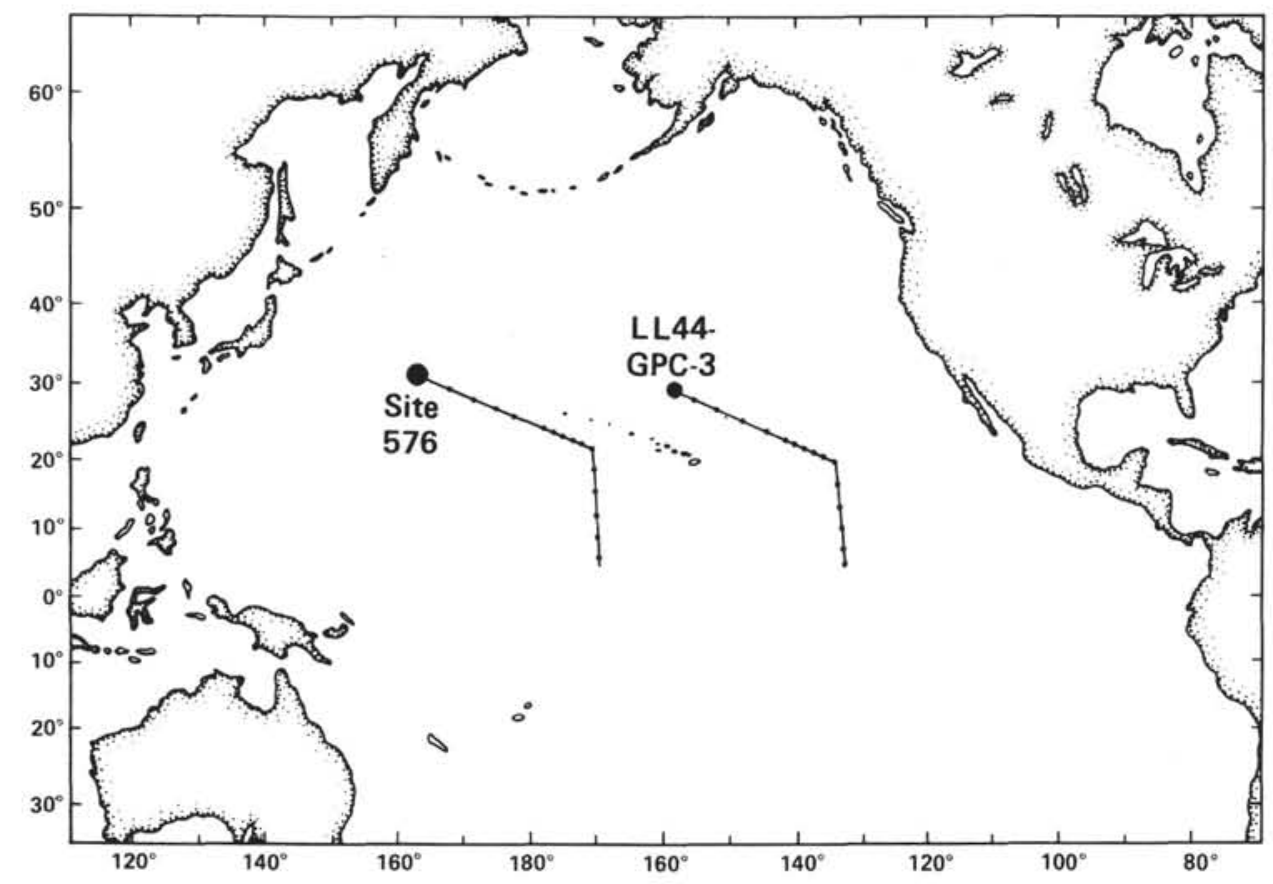

Figure 7. Backtrack path of DSDP Site 576 and LL44-GPC-3 (Leinen and Heath, 1981). Points on the backtrack path mark $5 \mathrm{~m}$.y. intervals along the path. 
ern Hemisphere glaciation. The high sedimentation rates at Site 578 allow increased resolution to evaluate better the changes in eolian input and atmospheric circulation with the onset of Northern Hemisphere glaciation.

Until recently, the onset of major Northern Hemisphere glaciation was thought to have occurred around $3.2 \mathrm{~m}$.y. ago. Much of the evidence supporting this date has come from low-resolution rotary-drilled DSDP cores and widely spaced samples. The inception of ice-rafting observed in rotary-drilled cores in the North Atlantic (DSDP Sites 111 and 116) was thought to occur about 3.0 m.y. ago (Berggren, 1972). In rotary-drilled cores in the Mediterranean Sea, Thunell (1979) found foraminiferal evidence for significant cooling around $3.2 \mathrm{~m} . \mathrm{y}$. ago. Oxygen-isotope analyses from foraminifers in the same sediments suggest a surface-water cooling at $3.2 \mathrm{~m}$.y. ago followed by an ice-volume increase between 3.1 and $3.0 \mathrm{~m} . y$. ago (Keigwin and Thunell, 1979). These results are in agreement with those of Saito (1976) and Keigwin (1979), which date the final closing of the Isthmus of Panama between 3.5 and 3.1 m.y. ago.

However, other evidence, including the eolian data presented here, suggest a later date for the onset of major ice buildup. In the Atlantic Ocean, off northwest Africa at Site 397, a significant increase in the variability of oxygen-isotope data occurs around $2.5 \mathrm{~m}$.y. ago (Shackleton and Cita, 1979). Herman (1974) recorded a distinct change in sediment characteristics and microfaunal abundances in the Arctic about 2.4 m.y. ago. More recent work (Shackleton et al., 1984) from Site 552 in the North Atlantic, shows that the onset of Northern Hemisphere glaciation, as marked by the appearance of an alternating sequence of nannofossil-foraminifer oozes and ice-rafted debris, occurred about 2.4 m.y. ago. A reexamination of the sediment from Sites 111 and 116 by Bachman (1979) resulted in an age of 2.5 m.y. for the earliest onset of ice-rafted debris at those sites.

Several data sets from the Pacific Ocean also suggest a later date for the onset of Northern Hemisphere glaciation. Conolly and Ewing (1970) recorded the beginning of ice-rafted debris about 2.5 m.y. ago from evidence in piston cores. Rea and Schrader (in press) came to a similar conclusion based upon the reexamination of ice-rafting in North Pacific cores. Shackleton and Opdyke (1977) analyzed oxygen isotopes in foraminifers in equatorial Pacific sediments which recorded a permanent change in isotopic composition around 3.1 m.y. ago. However, they also showed a major change in the variability of the oxygen isotopes around $2.5 \mathrm{~m} . \mathrm{y}$. ago, indicating glacial ice volume of a magnitude at least two-thirds that of the later Pleistocene glaciations.

The eolian mass-accumulation rates and grain size at Site 578 support the later date of 2.4 to $2.5 \mathrm{~m}$.y. ago for the onset of major ice buildup in the Northern Hemisphere. At Site 578, the eolian accumulation rates increase by more than a factor of two and appear to become highly variable around $3.0 \mathrm{~m}$.y. ago, near the time previously associated with the onset of Northern Hemisphere glaciation. However, following this temporary in- crease, eolian input decreases between 2.9 and 2.5 m.y. ago, then increases sharply at $2.5 \mathrm{~m}$.y. ago, and remains high and variable until the present. A similar pattern is seen in the eolian record at Sites 310 and 503 in the North Pacific. The accumulation rates at these two sites record a relative maximum around 2.9 to $2.75 \mathrm{~m}$.y. ago and an increase to higher rates around $2.5 \mathrm{~m}$.y. ago (Janecek, 1983).

This later age for the major climate change is also supported by the grain-size records at Sites 578, 310, and 503 (Janecek, 1983). All three sites record the largest grain size and hence wind vigor after $2.5 \mathrm{~m}$.y. ago. In addition, the grain-size record at Site 578 also suggests a maximum in wind intensity occurred around 1.5 to 1.0 m.y. ago.

Combined, these data suggest that the significant change in Northern Hemisphere continental climate occurred as late as 2.5 to $2.4 \mathrm{~m}$.y. ago. The increased eolian accumulation apparently is the result of the general increase in global aridity associated with stronger surface outflow of air from the continents, which enhances sinking motions and thus reduces rainfall (Manabe and Hahn, 1977). The changes in grain size would be a natural consequence of large ice expansion in the Northern Hemisphere as albedo feedback at high latitudes would result in steepened thermal gradients, increased wind intensity (Gates, 1976; Berger et al., 1981; CLIMAP, 1981), and hence larger grains transported to the deep sea.

The eolian records at Sites 578, 503, and 310 suggest that a "cooling event" or dry period may have occurred about 2.9 to $3.0 \mathrm{~m}$.y. ago. The eolian accumulation records at all three sites record peaks in accumulation at this time and suggest dryer (colder?) conditions in Asia, central America, and northern South America. The eolian grain size, however, does not change significantly at this time, suggesting the dryer (colder?) conditions were not accompanied by an increase in the intensity of atmospheric circulation.

Recent studies of oxygen isotopes in the Mediterranean and South Atlantic also suggest that a temporary global cooling occurred around $3.0 \mathrm{~m} . \mathrm{y}$. ago, prior to the onset of Northern Hemisphere glaciation. Isotopic records from the Mediterranean (Thunell and Williams, 1983) show that the interval between 4.2 and $2.5 \mathrm{~m}$.y. ago was marked by relatively warm and stable conditions. However, a short-term enrichment of ${ }^{18} \mathrm{O}$ occurred about 3.2 m.y. ago, which Thunell and Williams (1983) interpreted to reflect a cooling of surface water rather than a buildup of continental ice. A permanent shift in ${ }^{18} \mathrm{O}$ is recorded in the Mediterranean at $2.5 \mathrm{~m} . \mathrm{y}$. ago, indicating the initial large buildup of permanent continental ice (Thunell and Williams, 1983).

Isotopic records from the South Atlantic (Hodell et al., 1983) indicate no ${ }^{18} \mathrm{O}$ enrichment in the planktonic foraminifers at $3.2 \mathrm{~m}$.y. ago, although each site studied contained an enrichment in the benthic foraminifers that increased in magnitude with depth. Hodell et al., (1983) suggested that the event $3.2 \mathrm{~m}$.y. ago does not necessarily represent an increase in ice volume but rather a fun- 
damental change in deep-water ocean circulation patterns resulting from a global cooling event and/or the closing of the Central American Seaway.

\section{Implications for Long-Distance Transport}

The late Pleistocene eolian records at Sites 576 and 578 can be compared with other late Pleistocene North Pacific records from similar latitudes to constrain models of long-distance dust transport. Late Pleistocene eolian accumulation and grain size records from Sites 576 and 578 (this manuscript and unpublished data), University of Hawaii piston Core KK75-02 (Janecek and Rea, in press), and LL44-GPC-3 (Janecek and Rea, 1983) were averaged and plotted versus longitudinal distance (Fig. 8 ) to determine trends in accumulation and grain size with distance of transport. The reader is referred to the
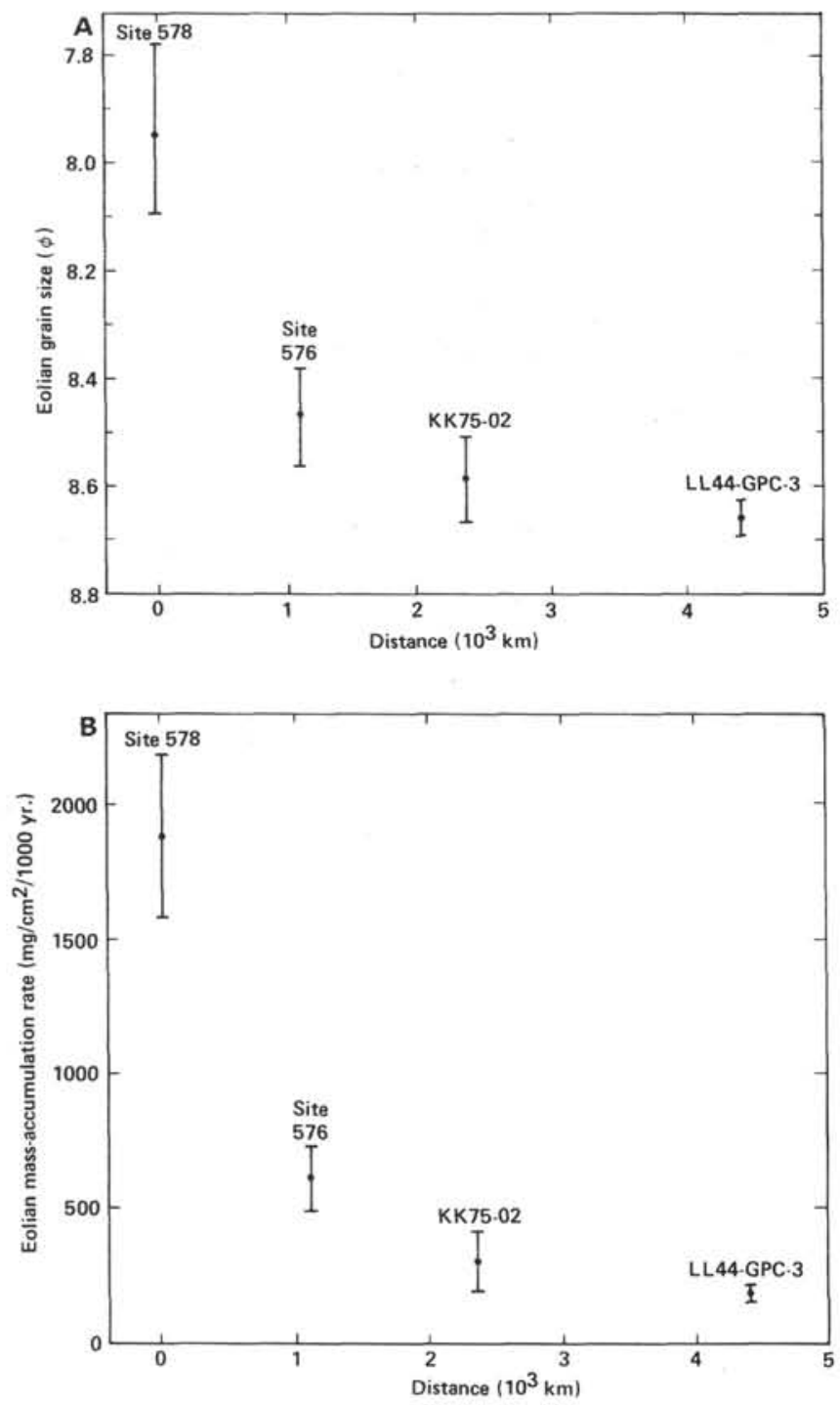

Figure 8. Distance of transport versus (A) eolian grain size and (B) eolian mass-accumulation rate for late Pleistocene samples from Site 578 ( 71 samples, this manuscript), Site 576 (64 samples, unpublished data), KK75-02 (90 samples, Janecek and Rea, in press), and LL44-GPC-3 (3 samples, Janecek and Rea, 1983). Error bars indicate one standard deviation. aforementioned references for a compilation and a more comprehensive analysis of the data for these cores. Both the accumulation rates and grain size decrease sharply between Sites 578 and 576 and then decrease very slowly farther to the east between Site 576 and LL44-GPC-3. This information suggests that the eolian material reaching Site 578 is influenced to a large extent by gravitational settling and thus records more and larger material. By the time that the eolian grains reach Site 576 in the central North Pacific, they have a size distribution that is nearly in equilibrium with the transporting wind. That is, the settling velocity of the grains is balanced by the lifting effects of atmospheric motions (Gillette et al., 1974; Gillette, 1981) and the grain size remains constant with further transport.

Recent work by Dauphin (1983) on quartz grains in the North Pacific has shown that there is a linear decrease in quartz size across the North Pacific. This implies a heterogeneous or continually changing mineral aerosol across the North Pacific. The grain-size and accumulation-rate data presented here, as well as the uniformity of quartz concentration between Site 576 and LL44-GPC-3 (Leinen, this volume), suggest that a very homogeneous dust composition is present after several thousand kilometers of transport. The data in Figure 8 suggest that Site 576 is not quite at the equilibrium point, which might explain why the grain size and accumulation rates for Site 576, while it has been under the influence of the westerlies, are greater than those of LL44GPC-3.

\section{Intensity of Atmospheric Circulation}

The atmospheric motions that support this equilibrium grain size can be quantified by using Stokes's settling law to calculate the settling velocity of dust grains in the air. Thus, changes in wind intensities in the past can be determined by comparing, in ratio form, the settling velocities calculated from the grain size from two different samples. As all terms except the grain size in Stoke's law will remain constant, the ratio of wind intensities between two different time horizons is given by the ratio of the squares of the larger, $D_{\mathrm{L}}^{2}$, and smaller, $D_{\mathrm{S}}{ }^{2}$, grain diameters (Janecek and Rea, in press).

These ratios can be applied to grain-size data sets from sites far enough from the source for grain size to be nearly in equilibrium with the transporting wind. Application of this ratio to the eolian grain-size record at Site 576 suggests that atmospheric circulation was reduced by at least a factor of 2.2 across the Paleocene/ Eocene boundary. Increases in wind intensity in the Oligocene and Pliocene are by factors of 1.8 and 1.4 , respectively. In comparison, at LL44-GPC-3 wind intensity decreases by a factor of 3.8 at the Paleocene/Eocene boundary. At this same site, wind intensity increases by a factor of 1.5 for the Oligocene, 1.8 for the Miocene, and 1.3 for the Pliocene.

\section{SUMMARY}

The grain size and mass-accumulation rate of eolian material isolated from Sites 576 and 578 in the Northwest Pacific have been used to evaluate changes in eolian 
sedimentation rate and the intensity of atmospheric circulation that have occurred during the past 70 m.y. The similarity in source regions and latitudinal positions of DSDP Site 576 and LL44-GPC-3 (located 3500 kilometers to the east) provides an opportunity to evaluate differences in long-distance transport of dust to the North Pacific during the Cenozoic. The temporal pattern of eolian accumulation rate and grain size is very similar in both cores. The eolian accumulation rate, an indicator of source area climate, is relatively low in the Late Cretaceous and early Cenozoic, in agreement with the humid conditions that characterized that time period. Increased eolian accumulation occurred at 25 m.y. ago, $15-10$ m.y. ago, and around 3.0 m.y. ago. The increase around $25 \mathrm{~m}$.y. ago is probably the result of the two sites moving into the westerlies, whereas the increase 15-10 m.y. ago coincides with the expansion of ice in Antarctica and a general increase in global aridity. An order of magnitude increase in accumulation rates in the Pliocene coincides with the onset of Northern Hemisphere glaciation. Eolian grain size, an indicator of wind intensity, suggests that Late Cretaceous and Paleocene time are characterized by wind intensities comparable to the present, a scenario supported by recent computer models of atmospheric circulation. A large decrease in grain size at the Paleocene/Eocene boundary is not readily explainable but suggests that a significant decrease in the intensity of atmospheric circulation may have occurred at that time. The lowest grain-size values occur in the middle Cenozoic and are in agreement with other proxy indicators of climate suggesting low thermal gradients and sluggish atmospheric circulation. The grain-size records indicate that relatively larger increases in atmospheric circulation occurred in the Oligocene (38 to 25 m.y. ago) and Miocene ( 20 to 10 m.y. ago) than with the onset of Northern Hemisphere glaciation in the Pliocene.

High-resolution samples from Site 578 record a temporary increase in grain size and accumulation rate around 3.0 m.y. ago and a permanent increase in both parameters $2.5 \mathrm{~m}$.y. ago. These records lend support to the idea that Northern Hemisphere glaciation occurred 2.4 to 2.5 m.y. ago and that the earlier event (3.0 m.y. ago) was a temporary global cooling.

Differences in accumulation rate and grain size with transport distance suggest that these indicators decrease exponentially, not linearly, with transport distance across the North Pacific and do not reach an equilibrium state until at least $3000 \mathrm{~km}$ distance from source areas.

\section{ACKNOWLEDGMENTS}

This chapter represents work the author conducted while at the University of Michigan. This manuscript benefitted from discussions with David K. Rea and Joseph Morley. In addition, the manuscript was reviewed by Margaret Leinen and an anonymous reviewer who provided helpful suggestions for its improvement. This work was supported, in part, by the National Science Foundation, Grant OCE8024368.

\section{REFERENCES}

Bachman, J., 1979. Pliocene biostratigraphy of DSDP Sites 111 and 116 from the North Atlantic Ocean and the age of northern hemisphere glaciation. Stockholm Contrib. Geol., 32:113-137.
Bandy, O. L., Butler, E. A., and Wright, R. C., 1969. Alaskan upper Miocene marine glacial deposits and the Turborotalia pachyderma datum plane. Science, 166:607-609.

Barron, E. J., and Washington, W. M., 1982. The atmospheric circulation during warm geologic periods: Is the equator to pole surface temperature gradient the controlling factor? Geology, 10:633-636.

Berger, W. H., 1976. Biogenous deep sea sediments: Production preservation and interpretation. In Riley, J. P., and Chester, R. (Eds.), Chemical Oceanography (Vol. 5): New York (Academic Press), 265-388.

Berger, W. H., Vincent, E., and Theirstein, H. R., 1981. The deep-sea record: Major steps in Cenozoic ocean evolution. In Warme, J. E., Douglas, R. G., and Winterer, E. L. (Eds.), The Deep Sea Drilling Project: A Decade of Progress. Soc. Econ. Paleontol. Mineral. Spec. Publ., 32:489-504.

Berggren, W. A., 1972. Late Pliocene-Pleistocene glaciation. In Laughton, A. S., Berggren, W. A., et al., Init. Repts. DSDP, 12: Washington (U.S. Govt. Printing Office), 953-963.

Brewster, N. A., 1980. Cenozoic biogenic silica sedimentation in the Antarctic Ocean. Geol. Soc. Am. Bull., 91(1):337-347.

CLIMAP Project Members, 1981. Seasonal Reconstruction of the Earth's Surface at the Last Glacial Maximum. Map and Chart Series 36 (Text, Map, and Microfiche), Geol. Soc. Am., Boulder, Colorado.

Conolly, J. R., and Ewing, M., 1970. Ice-rafted detritus in northwest Pacific sediments. In Hays, J. D. (Ed.), Geological Investigation of the North Pacific. Mem. Geol. Soc. Am., 126:219-232.

Dauphin, J. P., 1983. Eolian quartz granulometry as a paleowind indicator in the northeast Atlantic, North Pacific, and southwest equatorial Pacific [Ph. D. dissert.]. University of Rhode Island, Kingston.

Duce, R. A., Unni, C. K., Ray, B. J., Prospero, J. M., and Merrill, J. T., 1980. Long-range atmospheric transport of soil dust from Asia to the tropical North Pacific: Temporal variability. Science, 209: $1522-1524$.

Frakes, L. A., 1979. Climates Throughout Geologic Time: New York (Elsevier).

Gates, W. L., 1976. Modelling the ice age climate. Science, 191: $1138-1144$.

Gillette, D. A., 1981. Production of dust that may be carried great distances, In Pewe, T. L. (Ed.), Desert Dust: Origin, Characteristics, and Effect on Man. Geol. Soc. Am. Spec. Pap., 186:11-26.

Gillette, D. A., Blifford, I. H., Jr., and Fryrear, D. W., 1974. The influence of wind velocity on the size distributions of aerosols generated by wind erosion of soils. J. Geophys. Res., 79:4068-4075.

Glaccum, R. A., and Prospero, J. M., 1980. Saharan aerosols over the tropical North Atlantic-mineralogy. Mar. Geol., 37:295-321.

Graham, E. J., and Rea, D. K., 1980. Grain size and mineralogy of sediment cores from western Lake Huron. J. Great Lakes Res., 6: 129-140.

Herman, Y., 1974. Arctic Ocean sediments, microfauna, and the climatic record of the late Cenozoic, In Herman, Y. (Ed.), Marine Geology and Oceanography of the Arctic Sea: New York (Springer-Verlag), pp. 283-348.

Hodell, D. A., Williams, D. F., and Kennett, J. P., 1983. Fundamental changes in deep and intermediate water circulation patterns in the Western South Atlantic at $3.2 \mathrm{Ma}$. Geol. Soc. Am. Abstracts with Program, 15:596.

Honjo, S., 1983. Transport mechanism of pelagic particles in time and space. Trans. Am. Geophys. Union, 65:225.

1980. Material fluxes and modes of sedimentation in the mesopelagic and bathypelagic zones. J. Mar. Res., 38:53-97.

Jackson, M. L., Gillette, D. A., Danielson, E. F., Blifford, I. H., Bryson, R. A., and Syers, J. K., 1973. Global dustfall during the Quaternary as related to environments. Soil Sci., 116:135-145.

Janecek, T. R., 1983. The history of eolian sedimentation and atmospheric circulation during the Late Cenozoic [Ph. D. dissert.]. University of Michigan, Ann Arbor.

Janecek, T. R., and Rea, D. K., 1983. Eolian deposition in the northeast Pacific Ocean: Cenozoic history of atmospheric circulation. Geol. Soc. Am. Bull., 94:730-738.

in press. Quaternary fluctuations in the northern hemisphere westerlies and tradewind. Quat. Res.

Johnson, L. R., 1976. Particle-size fractionation of eolian dusts during transport and sampling. Mar. Geol., 21:M17-M21. 
1979. Mineralogical dispersal patterns of North Atlantic deep-sea sediments with particular reference to eolian dust. Mar. Geol., 29:335-345.

Keigwin, L. D., 1979. Late Cenozoic stable isotope stratigraphy and paleoceanography of DSDP sites from the east equatorial and north central Pacific Ocean. Earth Planet. Sci. Lett., 45:361-382.

Keigwin, L. D., and Thunnell, R. 1979. Middle Pliocene climatic change in the western Mediterranean from faunal and oxygen isotopic trends. Nature (London), 282:294-296.

Kemp, E. M., 1978. Tertiary climatic evolution and vegetation history in the southeast Indian Ocean region. Palaeogeogr., Palaeoclimatol., Palaeoecol., 24:169-208.

Kennett, J. P., and Shackleton, N. J., 1979. Oxygen isotopic evidence for the development of the psychrosphere 38 mya. Nature (London). 260:169-208.

Kolla, V., Biscaye, P. E., and Hanley, A. F., 1979. Distribution of quartz in late Quaternary Atlantic sediments in relation to climate. Quat. Res., 11:261-277.

Leinen, M., and Heath, G. R., 1981. Sedimentary indicators of atmospheric circulation in the Northern Hemisphere during the Cenozoic. Palaeogeogr., Palaeoclimatol., Palaeoecol., 36:1-21.

Manabe, S., and Hahn, D. C., 1977. Simulation of the tropical climate of the ice age. J. Geophys. Res., 82:3889-3912.

Molina-Cruz, A., and Price, P., 1977. Distribution of opal and quartz on the ocean floor of the subtropical southeastern Pacific. Geology, 5:81-84.

Parkin, D. W., 1974. Trade winds during the glacial cycles. Proc. $R$. Soc. London, Ser. A, 337:73-100.

Parkin, D. W., and Padgham, R. C., 1975. Further studies on trade winds during the glacial cycles. Proc. $R$. Soc. London Ser. A, 346: 245-260.

Pisias, N. G., and Leinen, M., 1984. Milankovitch forcing of the oceanic system: evidence from the Northwest Pacific. In Berger, A., and Imbrie, J. (Eds.), Milankovitch and Climate, Part 1: Dordrecht (Reidel), pp. 307-330.

Prospero, J. M., and Bonatti, E., 1969. Continental dust in the atmosphere of the eastern Equatorial Pacific. J. Geophys. Res., 74: 3362-3371.

Prospero, J. M., Glaccum, R. A., and Nees, R. T., 1981. Atmospheric transport of soil dust from Africa to South America. Nature (London), 289:570-572.

Prospero, J. M., and Nees, R. T., 1977. Dust concentrations in the atmosphere of the equatorial North Atlantic; Possible relationships to the Sahalian droughts. Science, 196:1196-1198.

Rea, D. K., and Janecek, T. R., 1981a. Late Cretaceous history of eolian deposition in the Mid-Pacific Mountains, central North Pacific Ocean. Palaeogeogr., Palaeoclimatol., Palaeoecol., 36:55-67. 1981b. Mass accumulation rates of the non-authigenic, inorganic, crystalline (eolian) component of deep sea sediments from the western Mid-Pacific Mountains, DSDP Site 463. In Thiede, J., Vallier, T. L., et al., Init. Repts. DSDP, 62: Washington (U.S. Govt. Printing Office), 653-659.

1982. Late Cenozoic changes in atmospheric circulation deduced from North Pacific eolian sediments. Mar. Geol., 49: 149-167.
Rea, D. K., and Schrader, H., in press. Late Pliocene onset of glaciation: Ice-rafting and diatom stratigraphy of North Pacific DSDP cores. Palaeogeogr., Palaeoclimatol., Palaeoecol.

Rex, R. W., and Goldberg, E. D., 1958. Quartz content of pelagic sediments of the Pacific Ocean. Tellus, 10:153-159.

Saito, T., 1976. Geological significance of coiling direction in Planktonic foraminifera, "Pulleniatina." Geology, 4:305-309.

Savin, S. M., 1977. The history of the earth's surface temperature during the past 100 million years. Ann. Rev. Earth Planet. Sci., 5: 319-335.

Shackleton, N. J., Backman, J., Zimmerman, H. B., Kent, D., Hall, M. A., Roberts, D. G., Schnitker, D., Baldauf, J. G., Despraries, A., Homringhausen, R., Huddlestun, P., Keene, J. B., Kaltenback, A. J., Krumsiek, K. A. O. Morton, A. C., Murray, J. W., and Westberg-Smith, J., 1984. Oxygen isotope calibration of the onset of ice-rafting and history of glaciation in the North Atlantic region. Nature (London), 307:620-623.

Shackleton, N. J., and Cita, M. B., 1979. Oxygen and carbon isotope stratigraphy of benthic foraminifers at Site 397: detailed history of climatic change during the Late Neogene. In von Rad, U., Ryan, W. B. F., et al., Init. Repts. DSDP, 47: Washington (U.S. Govt. Printing Office), 433-444.

Shackleton, N. J., and Opdyke, N. D., 1977. Oxygen isotope and paleomagnetic evidence for early Northern Hemisphere glaciation. Nature (London), 270:216-219.

Schultz, L., 1979. Saharan dust transport over the Atlantic, model calculations and measurements. In Morales, C. (Ed.), Saharan Dust Mobilization, Transport and Deposition: New York (Wiley), pp. 267-277.

Thiede, J., 1979. Wind regimes over the Late Quaternary southwest Pacific Ocean. Geology, 7:259-262.

Thunell, R. C., 1979. Pliocene-Pleistocene paleotemperatures and paleosalinity history of the Mediterranean Sea, results from Deep Sea Drilling Project Sites 125 and 132. Mar. Micropaleontol., 4: 173-177.

Thunell, R. C., and Williams, R. F., 1983. The stepwise development of Pliocene-Pleistocene paleoclimatic conditions in the Mediterranean based on high resolution oxygen isotopic studies. Geol. Soc. Am. Abstracts with Program, 15:706.

Windom, H. L., 1969. Atmospheric dust records in permanent snow fields: Implications to marine sedimentation. Geol. Soc. Am. Bull., 80:761-782.

, 1975. Eolian contributions to marine sediments J. Sediment. Petrol., 45:520-529.

Wolfe, J. A., 1978. A paleobotanical interpretation of Tertiary climates in the northern hemisphere. Am. Sci., 66:694-703.

Wolfe, J. A., and Hopkins, D. M., 1967. Climatic changes recorded by Tertiary land floras in northwestern North America. In Hatai, $\mathrm{K}$. (Ed.), Tertiary Correlations and Climatic Changes in the Pacific. Pacific Science Congress, 11th, Tokyo, Symposium 25:67-76.

Woodruff, F., Savin, S. M., and Douglas, R. G., 1981. Miocene stable isotope record: A detailed deep Pacific Ocean study and its paleoclimatic implications. Science, 212:665-668.

Date of Initial Receipt: 23 October 1983

Date of Acceptance: 20 August 1984 